\title{
Soliton Quantization in Lattice Field Theories
}

\author{
J. Fröhlich ${ }^{1}$ and P. A. Marchetti ${ }^{2}$ \\ 1 Theoretical Physics, ETH-Hönggerberg, CH-8093 Zürich, Switzerland \\ 2 Dipartimento di Fisica, Università di Padova, I.N.F.N. sez. Padova, I-35100 Padova, Italy
}

\begin{abstract}
Quantization of solitons in terms of Euclidean region functional integrals is developed, and Osterwalder-Schrader reconstruction is extended to theories with topological solitons. The quantization method is applied to several lattice field theories with solitons, and the particle structure in the soliton sectors of such theories is analyzed. A construction of magnetic monopoles in the fourdimensional, compact $U(1)$-model, in the QED phase, is indicated as well.
\end{abstract}

\section{Introduction}

In this paper we present a Euclidean method for the quantization of solitons and give an outline of the particle structure analysis on soliton sectors in massive lattice field theories. A discussion of monopoles and charged states in massless, abelian field theories has been presented in a separate paper [1], but the main findings related to monopoles are reviewed in Sect. 6 . While the main ideas also apply to continuum theories, we restrict our attention, in the more technical sections, to the lattice approximation, where our general approach can be implemented with mathematical precision. Since we study low-energy behaviour of quantum field theory, the presence of an ultraviolet cutoff, such as a lattice, can be expected to be immaterial.

Our approach to soliton quantization is an elaboration of a proposal in [2] (see also [3] for related ideas), combined with the particle structure analysis of [4]. The basic idea is to construct Euclidean Green functions of soliton fields as expectations of suitable disorder operators obtained by coupling the theory to a (generalized) external gauge field whose curvature is concentrated in points. A similar approach to soliton quantization in two-dimensional models has been discussed in [5].

The main purpose of our paper is to show that, in the Euclidean approach, a unified analysis of quantum solitons, including their particle structure, can be carried out for a large variety of models. Kinks, vortices and magnetic monopoles are among the examples covered by our methods. 
1.1. General Characterization of Soliton Sectors. A classical soliton is a topologically stable, finite-energy solution of the Hamiltonian equations of motion of a classical field theory.

In quantum field theory, solitons appear as new superselection sectors, $\mathscr{H}_{q}$, in the Hilbert space, $\mathscr{H}$, of physical states, which are labelled by a topological charge and are orthogonal to the vacuum sector, $\mathscr{H}_{0}$. Thus

$$
\mathscr{H}=\bigoplus_{q} \mathscr{H}_{q},
$$

where $q$ is the eigenvalue of the topological charge on $\mathscr{H}_{q}$. All sectors, $\mathscr{H}_{q}$, are invariant under an algebra of local observables which when applied to the vacuum generates $\mathscr{H}_{0}$. The quantized soliton field will turn out to be an operator which maps the vacuum sector onto a soliton sector.

Soliton sectors carry a continuous unitary representation of space-time translations, whose generators, $P^{\mu}$, satisfy the relativistic spectrum condition

$$
\operatorname{spec} P^{0} \geqq 0, \quad \operatorname{spec} P^{\mu} P_{\mu} \geqq 0,
$$

and, for $q \neq 0, \mathscr{H}_{q}$ does not contain any translation-invariant vectors, (i.e. there are no vacua in $\mathscr{H}_{q}$ ). One cannot, in general, require a representation of the full Poincare group on the soliton sectors, at least in massless theories. For example, one may show that the Lorentz group cannot be unitarily implemented on the monopole sectors of a four-dimensional gauge theory with an unbroken $U(1)$ (even if the continuum limit of its lattice approximation exists) [1]. This situation would be met in the analysis of 't Hooft-Polyakov monopole sectors [5] in non-abelian gauge theories.

The spectral condition (1.1) is, however, enough for a particle interpretation of the states, as shown in [6].

If the theory is massive one expects that the state obtained by applying a soliton operator of charge 1 to the vacuum is a stable one-particle state, and that soliton sectors carry a unitary representation of the full Poincare group. We analyze the particle structure of soliton sectors in the lattice approximation for several different models in Sect. 4.

So far, the most detailed (heuristic) analysis of quantum solitons has been based on semi-classical approximations, where the classical soliton appears as a local minimum of the Hamiltonian, and one expands in quantum fluctuations around the classical field configuration [7]. Our analysis does not involve any approximations of this kind and is mathematically rigorous.

A different mathematically precise construction of soliton sectors has earlier been proposed, for two-dimensional models, using the Hamiltonian approach to quantum field theory and operator techniques in $[8,9]$. In contrast, the methods developed in this paper involve the study of Euclidean-region functional integrals.

1.2. Euclidean Construction of Solitons. Our approach to soliton quantization is based on constructing Euclidean Green functions for soliton fields in terms of functional expectations of order- and disorder-fields [10]. To fix ideas, we first describe its main features for the simple example of the two-dimensional Ising model. For this example, disorder fields have been introduced by Kadanoff and 
Ceva, [10]. In their paper the expectation value, $\langle(\cdot)\rangle$, of a bi-local disorder field $D\left(x_{1}, x_{2}\right)$ is defined by

$$
\left\langle D\left(x_{1}, x_{2}\right)\right\rangle=\frac{Z\left(C_{x_{1} x_{2}}\right)}{Z}
$$

Here $Z$ is the partition function of the 2-D Ising model; $C_{x_{1} x_{2}}$ is a curve in the dual lattice, joining the sites $x_{1}$ and $x_{2}$, and $Z\left(C_{x_{1} x_{2}}\right)$ is a modified partition function given by

$$
Z\left(C_{\lambda_{1} \lambda_{2}}\right)=\sum_{\left\{\sigma_{x}\right\}} \prod_{\langle x y\rangle \notin\left(C_{\left.\chi_{1} x_{2}\right)^{*}}\right.} e^{-\beta / 2\left(\sigma_{x}-\sigma_{y}\right)^{2}} \times \prod_{\langle x y\rangle \in\left(C_{\gamma_{1} x_{2}}\right)^{*}} e^{-\beta / 2\left(\sigma_{x}+\sigma_{y}\right)^{2}},
$$

where $\sigma$ is the spin field, and $*$ denotes passage to the dual cells.

One can view $Z\left(C_{x_{1} x_{2}}\right)$ as the partition function of the Ising model coupled to a $\mathbb{Z}_{2}$-valued external gauge field, $\omega$, with support on $\left(C_{x_{1} x_{2}}\right)^{*}$, [5]. In the representation of the Ising model in terms of Peierls contours, $Z\left(C_{x_{1} x_{2}}\right)$ is given by a sum over configurations of contours which contain, besides the usual closed contours, $\gamma_{i}$, an open contour, $\gamma_{x_{1} x_{2}}$, joining $x_{1}$ to $x_{2}$. Denoting the Boltzmann weight of a configuration $\{\gamma\}$ of contours by $Z(\{\gamma\})$, we can write

$$
\left\langle D\left(x_{1}, x_{2}\right)\right\rangle=\frac{\sum_{\left\{\gamma_{1}, \ldots, \gamma_{n}, \gamma_{x_{1}}, x_{2}\right\}} Z\left(\gamma_{1}, \ldots, \gamma_{n}, \gamma_{x_{1}, x_{2}}\right)}{\sum_{\left\{\gamma_{1}^{\prime}, \ldots, \gamma_{m}^{\prime}\right\}} Z\left(\gamma_{1}^{\prime}, \ldots, \gamma_{m}^{\prime}\right)} .
$$

This disorder correlation function turns out to be the Euclidean two-point function of the soliton field operator in the $2-D$ Ising model. We now recall the different possible behaviour of $\left\langle D\left(x_{1}, x_{2}\right)\right\rangle$ :

In the disordered phase, i.e. for small $\beta$, where Peierls contours condense, $\left\langle D\left(x_{1}, x_{2}\right)\right\rangle$ is bounded away from zero, uniformly in $\left|x_{1}-x_{2}\right|$, whereas in the ordered phase, i.e. for large $\beta$, where the Peierls contours form a dilute gas,

$$
\left\langle D\left(x_{1}, x_{2}\right)\right\rangle \underset{\left|x_{1}-x_{2}\right| \rightarrow \infty}{\longrightarrow} 0,
$$

exponentially fast.

The spin $\sigma$ is the usual "order parameter" for the Ising model, and the behaviour of the two-spin correlation function, $\left\langle\sigma_{x_{1}} \sigma_{x_{2}}\right\rangle$, is contrary to the one of $\left\langle D\left(x_{1}, x_{2}\right)\right\rangle$. This motivates calling $D\left(x_{1}, x_{2}\right)$ a disorder field.

Disorder fields can also be defined in the continuum limit and can be abstractly characterized in terms of topological singularities in the joint expectation values of order and disorder fields, as described in [2].

In this paper, we give an explicit construction of the disorder fields in a variety of models, closely following the one sketched above for the 2-D Ising model. We then show how expectation values of disorder fields are related to Green functions of soliton fields.

Generally speaking, the expectation value of a disorder field, $D(\omega)$, is given by

$$
\langle D(\omega)\rangle=\frac{Z(\omega)}{Z},
$$


where $\omega$ is a singular generalized external gauge field with values in a discrete abelian group, $\mathscr{Z} ; Z$ is the partition function of the theory, and $Z(\omega)$ denotes the partition function of the model coupled to the external field $\omega$.

If the support of the curvature, $d \omega$, of $\omega$ is a finite set of points, $\left\{x_{1}, \ldots, x_{n}\right\}$, with $(d \omega)^{*}\left(x_{i}\right)=q_{i} \in \mathscr{Z}$, then

$$
\langle D(\omega)\rangle \equiv\left\langle D\left(x_{1}, q_{1}, \ldots, x_{n}, q_{n}\right)\right\rangle
$$

turns out to be the Euclidean Green function, $S_{n}\left(x_{1} q_{1}, \ldots, x_{n} q_{n}\right)$, of soliton fields of charge $\left\{q_{i}\right\}$ located at $\left\{x_{i}\right\}, i=1,2, \ldots, n$.

In the Euclidean approach to the construction of vacuum sectors of a quantum field theory (Q.F.T.), the Hilbert space of states and the unitary representation of the Poincaré group are obtained by applying an Osterwalder-Schrader (O-S) reconstruction theorem to the Euclidean Green (correlation) functions of the order fields [11].

By applying an O-S reconstruction theorem to the entire set of Euclidean Green functions of order and soliton fields (i.e. to all joint order-disorder correlation functions) of a Q.F.T., all its soliton sectors can be recovered as well; (see Sect. 2.2). In fact, from the sequence of order-disorder correlation functions, soliton field operators, $S_{q}(x)$, with charge $q \in \mathscr{Z}$ can be reconstructed, and if cluster properties hold and the correlation functions with non-zero total charge vanish, then the resulting Hilbert space splits into sectors, $\mathscr{H}_{q}, q \in \mathscr{Z}$, and the soliton field operators, $S_{q}(x)$, map the vacuum sector $\mathscr{H}_{0}$ onto the soliton sectors $\mathscr{H}_{q}$.

We now rephrase this construction of soliton sectors in the language of defects; (for a definition of defects see e.g. [12]): Suppose that the partition function, $Z$, of some Euclidean field theory can be expressed as a sum over configurations of closed line defects, $v$, (analogues of the Peierls contours in the 2-D Ising model) carrying a charge $q$, with values in a discrete abelian group $\mathscr{Z}$, i.e.

$$
Z=\sum_{v . \partial v=0} Z(v)
$$

where $Z(v)$ is the Boltzmann weight of the configuration $v$. Then the correlation functions of the disorder fields are given by

$$
\left\langle D\left(x_{1}, q_{1}, \ldots, x_{n}, q_{n}\right)\right\rangle=\frac{1}{Z} \sum_{v: \partial v=\left\{x_{1}, q_{1}, \ldots, x_{n}, q_{n}\right\}} Z(v) .
$$

Let us assume that the correlation functions with non-zero total defect charge vanish, and clustering holds, so that, for example,

$$
\left\langle D\left(x_{1}, q_{1}, x_{2}, q_{2}\right)\right\rangle \rightarrow 0,
$$

as $\left|x_{1}-x_{2}\right| \nearrow \infty$.

Then the quantum field theory, reconstructed from the Euclidean field theory, has soliton sectors labelled by the (total) defect charge $q \in \mathscr{Z}, q \neq 0$, and these sectors can be obtained from the joint correlation functions of order- and disorder fields by analytic continuation in the time variables; (O-S reconstruction).

Let us denote by $\Omega, S_{q}, \phi, H$ the vacuum, the soliton field operator, the ordinary field operator and the Hamiltonian, respectively, obtained via $\mathrm{O}-\mathrm{S}$ reconstruction, 
and by $\Phi$ the Euclidean field corresponding to $\phi$, i.e. the order field. Then for $x_{1}^{0}<\ldots<x_{m}^{0}<y_{1}^{0}<\ldots<y_{n}^{0}$,

$$
\begin{aligned}
\langle\Phi( & \left.\left.x_{1}\right) \ldots \Phi\left(x_{m}\right) D\left(y_{1}, q_{1}, \ldots, y_{n}, q_{n}\right)\right\rangle \\
= & \left\langle\Omega, \phi\left(\mathbf{x}_{1}\right) e^{-\left(x_{2}^{0}-x_{1}^{0}\right) H} \phi\left(\mathbf{x}_{2}\right) \ldots e^{-\left(x_{m}^{0}-x_{m-1}^{0}\right) H} \phi\left(\mathbf{x}_{m}\right)\right. \\
& \left.\cdot e^{-\left(y_{1}^{0}-x_{m}^{0}\right) H} S_{q},\left(\mathbf{y}_{1}\right) \ldots e^{-\left(y_{n}^{0}-y_{n-1}^{0}\right) H} S_{q_{n}}\left(\mathbf{y}_{n}\right) \Omega\right\rangle .
\end{aligned}
$$

This equation corresponds to the usual Feynman-Kac formula. The three-point function

$$
\phi_{q}^{\varepsilon}(\mathbf{x}) \equiv\left\langle e^{-\varepsilon H} S_{q}(\mathbf{0}) \Omega, \phi(\mathbf{x}) e^{-\varepsilon H} S_{q}(\mathbf{0}) \Omega\right\rangle,
$$

with $\varepsilon>0$ small, has a behaviour reminiscent of the soliton solution with charge $q$, $\phi_{q}(\mathbf{x})$, of the corresponding classical field theory. (For more detailed comments see e.g. $[8,9]$.)

\section{Basic Definitions, and Classification of Solitons}

Since we shall show how our construction of quantum solitons works for lattice theories, we start by recalling some basic definitions concerning such theories. Formally, our methods extend to continuum theories, but then we loose rigorous mathematical control.

2.1. Lattice Field Theories. The lattice on which we work is $\mathbb{Z}_{1 / 2}^{d}$, $d$ being the dimension. A $k$-dimensional cell in the lattice is denoted by $c_{k}$; we also use the notations $c_{0}=x, c_{1}=\langle x y\rangle$ or $=b, c_{2}=p$. The symbol ${ }^{*}$, applied to cells, denotes passage to the dual cell; hence $c_{k}^{*}$ denotes a $(d-k)$-dimensional cell in the dual lattice, $\mathbb{Z}^{d}$, dual to $c_{k}$. If $W$ denotes a topological space, and $k$ is a positive integer, then a $W$-valued lattice field of rank $k$ is a map from the $k$-dimensional cells of the lattice to $W$.

The symbol * applied to a rank- $k$ field $\phi$, is the operation of passing to the dual field: $\phi^{*}$ is a field of rank $d-k$ on the dual lattice, defined by $\phi^{*}\left(c_{k}^{*}\right)=\phi\left(c_{k}\right)$. If $W$ is an abelian additive group one can define the lattice exterior differential, $d$, as a map from rank- $k$ to rank $-(k+1)$ lattice fields by setting

$$
d \phi\left(c_{k+1}\right)=\sum_{c_{k} \in \partial c_{k+1}} \phi\left(c_{k}\right)
$$

where $\partial$ denotes the boundary operation.

The lattice codifferential, $\delta$, is defined as a map from rank- $k$ to rank- $(k-1)$ fields, defined by

$$
\delta=* d^{*}
$$

Notice that $\delta^{2}=d^{2}=0$. The lattice Laplacian is defined by

$$
\Delta=d \delta+\delta d .
$$

If $W$ is a Hilbert space with scalar product $(\cdot, \cdot)_{W}$, then a scalar product on the space of $W$-valued fields of rank $k$ is defined by

$$
\left(\phi, \phi^{\prime}\right)=\sum_{c_{k}}\left(\phi\left(c_{k}\right), \phi^{\prime}\left(c_{k}\right)\right)_{W}
$$


If $W=\mathbb{R}$, then $\delta$ is the adjoint of $d$ with respect to the scalar product $(\cdot, \cdot)$.

We now describe different lattice field theories in more detail.

A scalar field $\phi$ is a map from the sites, $x$, to some vector space $V_{H}$, with norm $\|\cdot\|$. The vacuum functional of a scalar theory is given by

$$
d \mu(\phi)=\frac{1}{Z}\left(\prod_{x} d \phi_{x}\right) e^{-S(\phi)} G_{\mathrm{b} . \mathrm{c}}(\phi) .
$$

In (2.5), $d \phi_{x}$ is the Lebesgue measure on $V_{H}, Z$ is a partition function chosen such that

$$
\int d \mu(\phi)=1
$$

and $G_{\mathrm{bc}}$ specifies some boundary conditions. Finally, $S$ is the action:

$$
\begin{aligned}
S(\phi) & =S_{0}(\phi)+S_{1}(\phi), \\
S_{0}(\phi) & =\frac{\beta_{H}}{2} \sum_{\langle x y\rangle}\left\|d \phi_{\langle x y\rangle}\right\|^{2}, \\
S_{1}(\phi) & =\lambda \sum_{x} V\left(\phi_{x}\right),
\end{aligned}
$$

where $V$ is a positive function, and $\beta_{H}, \lambda$ are positive constants. $S_{0}$ is the kinetic term, and $S_{1}$ is the interaction term of the action. (To be rigorous, the measure should be defined first for a finite lattice, $\Lambda \subset \mathbb{Z}_{1 / 2}^{d}$, with $G_{\mathrm{bc}}$ depending on $\left\{\phi_{x}\right\}_{x \in \partial \Lambda}$. Afterwards one should take the limit $\Lambda \nearrow \mathbb{Z}_{1 / 2}^{d}$. See e.g. [12]. We omit henceforth any reference to the finite lattice $\Lambda$ and assume to work always in the thermodynamic limit, unless $\Lambda$ is explicitly mentioned.)

A Fermion field is an anticommuting map from the sites of the lattice to the orthonormal frames of a vector space $V_{S} \times V_{F}$, the Fermion space, where $V_{S}$, the spin space, carries a representation of the Dirac-Clifford algebra.

The vacuum functional of a Fermion theory interacting with an external scalar field, $\varphi$, is given by

$$
d \mu(\bar{\psi}, \psi)=\frac{1}{Z}\left(\prod_{x} d \bar{\psi}_{x} d \psi_{x}\right) e^{-S(\bar{\psi}, \psi, \phi)},
$$

where $d \bar{\psi}_{x} d \psi_{x}$ is the Berezin integration, and $S$ is the action, e.g.

$$
\begin{aligned}
S(\bar{\psi}, \psi, \phi) & =S_{0}(\bar{\psi}, \psi)+S_{1}(\bar{\psi}, \psi, \phi), \\
S_{0}(\bar{\psi}, \psi) & =\frac{\beta_{F}}{2} \sum_{\langle x y\rangle} \bar{\psi}_{x} \Gamma_{\langle x y\rangle} \psi_{y}, \\
S_{1} & =\lambda \sum_{x} \bar{\psi}_{x} P\left(\phi_{x}\right) \psi_{x} .
\end{aligned}
$$

In (2.8), $P(\phi)$ denotes a polynomial in $\phi$ with coefficients in the space of matrices acting on the Fermion space, and

$$
\Gamma_{\langle x y\rangle}=\underset{(-)}{+} \gamma_{\mu}, \quad \begin{aligned}
& \text { if }\langle x y\rangle \text { is in the positive } \\
& \text { (negative) } \mu \text {-direction, }
\end{aligned}
$$

where $r=0$ for "naive" Fermions, and $r=1$ for Wilson Fermions. 
A gauge field, $g$, is a map from the links of the lattice to a group $G$, the gauge group. Let $C$ be an oriented curve. We define

$$
g_{C}=\prod_{\langle x y\rangle \in C} g_{\langle x v\rangle},
$$

where $\vec{\prod}$ is the path ordered product. Moreover, let $\phi(\psi)$ be a scalar (Fermion) field, with $V_{H}\left(V_{F}\right)$ carrying a unitary representation $U_{H}\left(U_{F}\right)$ of $G$.

The vacuum functional of a gauge theory with matter fields $\phi, \psi$ is given by

$$
d \mu(g, \phi, \bar{\psi}, \psi)=\frac{1}{Z}\left(\prod_{\langle x y\rangle} d g_{\langle x y\rangle} \prod_{x} d \phi_{x} d \bar{\psi}_{x} d \psi_{x}\right) e^{-S(g, \phi, \bar{\psi}, \psi)},
$$

where $d g_{\langle x y\rangle}$ is the Haar measure on $G$, and $S$ is the total action, given by

$$
\begin{aligned}
S & =S_{0}(g)+S_{1}(g, \phi)+S_{1}(g, \bar{\psi}, \psi)+S_{1}(\phi), \\
S_{0}(g) & =\frac{\beta_{G}}{2} \sum_{p} \operatorname{Re}\left(\chi(1)-\chi\left(g_{\partial p}\right)\right), \\
S_{1}(g, \phi) & =\frac{\beta_{H}}{2} \sum_{\langle x y\rangle}\left\|\phi_{x}-U_{H}\left(g_{\langle x y\rangle}\right) \phi_{y}\right\|^{2}, \\
S_{1}(g, \bar{\psi}, \psi) & =\frac{\beta_{F}}{2} \sum_{\langle x y\rangle} \bar{\psi}_{x} \cdot \Gamma_{\langle x y\rangle} \otimes U_{F}\left(g_{\langle x y\rangle}\right) \psi_{y},
\end{aligned}
$$

where $\chi$ denotes a character of $G$.

If the gauge group is $\mathbb{R}$, then the gauge field is usually denoted by $A$ and the kinetic action $S_{0}$ is quadratic, i.e.

$$
S_{0}(A)=\frac{\beta_{G}}{2} \sum_{p}(d A)_{p}^{2}=\frac{\beta_{G}}{2}(d A, d A),
$$

with a local gauge fixing such as

$$
S_{\mathrm{fix}}(A)=\frac{1}{2 \alpha} \sum_{x}(\delta A)_{x}^{2}=\frac{1}{2 \alpha}(\delta A, \delta A) .
$$

A gauge theory with scalar matter fields is called a Higgs model.

If $G$ is abelian, one can define higher-rank gauge theories with matter fields, where the role of the gauge field is played by a field of $\operatorname{rank} k>1$, and the role of the matter field is played by a field of rank $k-1$.

In gauge theories, natural observables are given by gauge-invariant functions of the basic fields, such as the Wilson loop, $O(C)=\chi\left(g_{C}\right)$, or the string variables

$$
\begin{aligned}
O_{H}\left(C_{x y}\right) & =\left(\phi_{x}, \prod_{b \in C_{x v}} U_{H}\left(g_{b}\right) \phi_{y}\right), \\
O_{F}\left(C_{x y}\right) & =\bar{\psi}_{x} \cdot \underset{b \in C_{x y}}{\longrightarrow} U_{F}\left(g_{b}\right) \psi_{y},
\end{aligned}
$$

where $C$ is a loop, and $C_{x y}$ a path from $x$ to $y$. 
2.2. Reconstruction Theorem and Soliton Sectors. In this section we briefly review the basic reconstruction theorem, applied to joint order-disorder correlation functions of a lattice field theory and define precisely what we mean by a soliton sector of a lattice theory.

As shown in the next section, we are interested in correlation functions given by the v.e.v. of a disorder field $D\left(x_{1}, q_{1}, \ldots, x_{n}, q_{n}\right)$ and a monomial of gauge invariant functions of the basic fields, $O(C)$, with support on a compact connected set of cells, $C$, such as a loop or a string.

More precisely, we will consider correlation functions of the form

$$
S_{n, m}\left(x_{1}, q_{1}, \ldots, x_{n}, q_{n} ; C_{1}, \ldots, C_{m}\right)=\left\langle D\left(x_{1}, q_{1}, \ldots, x_{n}, q_{n}\right) O\left(C_{1}\right) \ldots O\left(C_{m}\right)\right\rangle,
$$

with $\sum_{i} q_{i}=0$. Correlation functions with non-vanishing total charge are defined by a limiting procedure, removing a charge to infinity; i.e., for $\sum_{i} q_{i}=q \neq 0$,

$$
\begin{aligned}
& S_{n, m}\left(x_{1}, q_{1}, \ldots, x_{n}, q_{n} ; C_{1}, \ldots, C_{m}\right) \\
& \quad \equiv C_{q} \lim _{z \rightarrow \infty} S_{n+1}\left(x_{1}, q_{1}, \ldots, x_{n}, q_{n}, z,-q ; C_{1}, \ldots, C_{m}\right)
\end{aligned}
$$

where $C_{q}$ is a normalization constant needed if $\lim _{x \rightarrow \infty} S_{2,0}(0, q, x,-q) \neq 0$, and then given by

$$
C_{q}=\left(\lim _{x \rightarrow \infty} S_{2,0}(0, q, x,-q)\right)^{-1 / 2} .
$$

The precise definition of $S_{n, m}$ for variety of lattice field theories will be given in Sect. 3.3.

We now turn to the reconstruction theorem. We define the $\theta$-operation as an antilinear map which acts on the basic fields of the theory as follows.

Let $r$ denote the reflection in the time-zero plane, $f$ a complex function, and $\bar{f}$ its complex conjugate. Then,

$$
\begin{aligned}
(\theta f)\left(\phi_{x}\right) & =\overline{f\left(\phi_{r x}\right)}, & (\theta f)\left(g_{\langle x y\rangle}\right) & =\overline{f\left(g_{\langle r x, r y\rangle}\right)}, \\
\theta \psi_{x} & =\bar{\psi}_{r x} \gamma_{0}, & \theta \bar{\psi}_{x} & =\gamma_{0} \psi_{r x} .
\end{aligned}
$$

Consider expectations of the form $\langle F \theta F\rangle$, where $F$ belongs to a linear space, $\mathscr{F}_{+}$, of functions of the basic fields over the positive-time lattice. These expectations can be expressed in terms of a sum over correlation functions $S_{n, m}$.

If for every $F$ in $\mathscr{F}_{+},\langle F \theta F\rangle>0$, then the correlation functions $\left\{S_{n, m}\right\}$ are said to be Osterwalder-Schrader (reflection) positive. The reconstruction theorem is the following result.

Theorem 2.1. If the set of correlation functions $\left\{S_{n, m}\right\}$ is

i) lattice-translation-invariant, and

ii) O-S positive, then one can reconstruct from $\left\{S_{n, m}\right\}$

a) a separable Hilbert space, $\mathscr{H}$, of physical states,

b) a vector $\Omega \in \mathscr{H}$ of unit norm, the vacuum, 
c) a selfadjoint transfer matrix $T$ with norm $\|T\| \leqq 1$, and unitary spatial translation operators $U_{\mu}, \mu=1, \ldots, d-1$, acting on $\mathscr{H}$, such that $T \Omega=U_{\mu} \Omega=\Omega$. If, moreover, the correlation functions $\left\{S_{n, m}\right\}$ satisfy

iii) cluster properties then

d) $\Omega$ is the unique vector in $\mathscr{H}$ invariant under $T$ and $U_{\mu}$.

Let us briefly sketch how this theorem is proven; (for more details see [11]).

First we construct the Hilbert space $\mathscr{H}$. We equip the space $\mathscr{F}_{+}$with a positive semi-definite inner product, $\langle\cdot, \cdot\rangle$, by setting

$$
\langle F, G\rangle=\langle(\theta F) G\rangle,
$$

for $F$ and $G$ in $\mathscr{F}_{+}$. Let $\mathscr{N}$ be the Kernel of $\langle\cdot, \cdot\rangle$, i.e. the subspace of $\mathscr{F}_{+}$of functions $F$, with $\langle F, F\rangle=0$.

We set $\mathscr{H}=\overline{\mathscr{F}}+/ \mathcal{N}$, where the closure is taken in the norm $\|F\|=\sqrt{\langle F, F\rangle}$. Given $F \in \mathscr{F}$, we denote by $\hat{F}$ the equivalence class of $F \bmod \mathscr{N}$, which is a vector in $\mathscr{H}$. The vacuum $\Omega$ is defined by $\Omega=\hat{1}$. We define $T(t) \equiv T^{t}$ by

$$
\langle\hat{F}, T(t) \hat{G}\rangle=\left\langle(\theta F) G_{t}\right\rangle,
$$

where $t \in \mathbb{Z}_{+}$, and $G_{t}$ denotes the translate of $G$ in the positive time direction by $t$ lattice units. By translation invariance

$$
\left\langle(\theta F) G_{t}\right\rangle=\left\langle(\theta F)_{-t} G\right\rangle=\left\langle\left(\theta F_{t}\right) G\right\rangle=\langle T(t) \hat{F}, \hat{G}\rangle,
$$

i.e., $T$ leaves $\mathcal{N}$ invariant and is symmetric. By the Schwarz inequality and the symmetry of $T$,

$$
\begin{aligned}
|\langle\hat{F}, T(t) \hat{G}\rangle| & \leqq\langle\hat{F}, \hat{F}\rangle^{1 / 2}\langle\hat{G}, T(2 t) \hat{G}\rangle^{1 / 2} \leqq \ldots \\
& \leqq\langle\hat{F}, \hat{F}\rangle^{1 / 2}\langle\hat{G}, \hat{G}\rangle\left(\sum_{n=2}^{N} 2^{-n}\right)\left\langle\hat{G}, T\left(2^{N} t\right) \hat{G}\right\rangle^{1 / 2 N}
\end{aligned}
$$

Since $\left\langle\theta G(G)_{2} N_{t}\right\rangle$ is bounded in $t$,

Hence

$$
\lim _{N \rightarrow \infty}\left\langle\hat{G}, T\left(2^{N} t\right) \hat{G}\right\rangle^{1 / 2 N}=1 .
$$

$$
|\langle\hat{F}, T(t) \hat{G}\rangle| \leqq\|\hat{F}\| \cdot\|\hat{G}\|,
$$

i.e. $\|T\| \leqq 1$, and hence $T$ is selfadjoint. We define $U(\mathbf{a})=\prod_{\mu=1}^{d=1} U_{\mu}^{a_{\mu}}$ by

$$
\langle\hat{F}, U(\mathbf{a}) \hat{G}\rangle=\left\langle(\theta F) G_{\mathbf{a}}\right\rangle \text {. }
$$

Now

$$
\left\langle(\theta F) G_{\mathbf{a}}\right\rangle=\left\langle(\theta F)_{\mathbf{a}} G\right\rangle=\left\langle\left(\theta F_{-\mathbf{a}}\right) G\right\rangle=\langle U(-\mathbf{a}) \hat{F}, \hat{G}\rangle,
$$

i.e. $U(\mathbf{a})$ is unitary. Clearly $T$ and $U(\mathbf{a})$ commute.

We remark that, since $\|T\| \leqq 1$ and the operators $U_{\mu}$ are unitary, we may apply the spectral theorem to obtain

$$
\langle\hat{F}, T(t) U(\mathbf{a}) \hat{F}\rangle=\int d \varrho_{F}(\lambda, \mathbf{k}) \lambda^{t} e^{i \mathbf{k} \cdot \mathbf{a}},
$$

for some positive measure $d \varrho_{F}$ with support in $[-1,1] x[-\pi, \pi]^{d-1}$. 
From the explicit proof of the theorem it follows that there is a set of vectors $\left\{\left|x_{1}, q_{1}, \ldots, x_{n}, q_{n} ; C_{1}, \ldots, C_{m}\right\rangle\right\}$ in $\mathscr{H}$, with $\left\{x_{i}\right\}$ in the positive-time dual lattice, and $\left\{C_{j}\right\}$ in the positive-time lattice, such that the set of their linear combinations, $\widehat{\mathscr{F}}_{+}$, is dense in $\mathscr{H}$. On these vectors the scalar product is defined by

$$
\begin{aligned}
\left\langle x_{1},\right. & q_{1}, \ldots, x_{n}, q_{n} ; C_{1}, \ldots, C_{m}\left|x_{1}^{\prime}, q_{1}^{\prime}, \ldots, x_{r}^{\prime}, q_{r}^{\prime} ; C_{1}^{\prime}, \ldots, C_{s}^{\prime}\right\rangle \\
= & \left\langle D\left(r x_{1},-q_{1}, \ldots, r x_{n},-q_{n}, x_{1}^{\prime}, q_{1}^{\prime} \ldots x_{r}^{\prime} q_{r}^{\prime}\right) .\right. \\
& \left.\cdot O\left(C_{1}^{\prime}\right) \ldots O\left(C_{s}^{\prime}\right) \theta\left[O\left(C_{1}\right) \ldots O\left(C_{m}\right)\right]\right\rangle .
\end{aligned}
$$

"Field operators", $A\left(x_{1}, q_{1}, \ldots, x_{n}, q_{n} ; C_{1}, \ldots, C_{m}\right)$, with $x_{i}$ and $C_{j}$ contained in the strip $\left\{x: x^{0} \in[0, t], t \in \mathbb{Z}+\right\}$, naturally act on $T(t) \hat{\mathscr{F}}_{+}$by setting

$$
\begin{aligned}
& \left.\left.A\left(x_{1}, q_{1}, \ldots, x_{n}, q_{n} ; C_{1}, \ldots, C_{m}\right) T(t) \mid x_{1}^{\prime}, q_{1}^{\prime}, \ldots, x_{r}^{\prime}, q_{r}^{\prime} ; C_{1}^{\prime}, \ldots, C_{s}^{\prime}\right)\right\rangle \\
& \quad=\left|x_{1}, q_{1}, \ldots, x_{n}, q_{n},\left(x_{1}^{\prime}\right)_{t}, q_{1}^{\prime}, \ldots,\left(x_{r}^{\prime}\right)_{t}, q_{r}^{\prime} ; C_{1}, \ldots, C_{m},\left(C_{1}^{\prime}\right)_{t}, \ldots,\left(C_{s}^{\prime}\right)_{t}\right\rangle,
\end{aligned}
$$

where $(\cdot)_{t}$ denotes translation by $t$ in the time direction. The operator $A(C)$ is called on order field operator and

$$
A(x, q) \equiv S_{q}(x)
$$

is called a soliton field operator of charge $q$.

We now define the superselection sectors. Let $\mathscr{A}$ denote the set of linear combinations of the field operators $A\left(x_{1}, q_{1}, \ldots, C_{m}\right)$, defined above, and $\mathscr{A}_{0}$ the subset of linear combinations of the field operators $A\left(C_{1}, \ldots, C_{m}\right)$. We call $\mathscr{A}$ the field algebra and $\mathscr{A}_{0}$ the observable algebra.

Definition 2.2. Suppose that the Hilbert space $\mathscr{H}$, obtained via reconstruction, decomposes into orthogonal sectors, $\mathscr{H}_{q}$, invariant under $T, U_{\mu}$ and $\mathscr{A}_{0}$, i.e. $\mathscr{H}=\bigoplus_{q} \mathscr{H}_{q}$. Define $\mathscr{H}_{0}^{\prime}$ to be the subspace given by

$$
\overline{\mathscr{A}_{0} \Omega}=\mathscr{H}_{0}^{\prime} ;
$$

$\mathscr{H}_{0}^{\prime}$ is called vacuum sector.

A sector $\mathscr{H}_{q} \perp \mathscr{H}_{0}^{\prime}$ is called a charged sector if there are no lattice-translationinvariant vectors in $\mathscr{H}_{q}$.

Charged sectors, $\mathscr{H}_{q}, q \neq 0$, are called soliton sectors if the charge, $q$, is a defect charge (as defined in Sect. 1.2).

If we denote by $\mathscr{A}_{\text {neutral }}$ the set of linear combinations of operators $\left\{A\left(x_{1}, q_{1}, \ldots, C_{m}\right): \sum_{i} q_{i}=0\right\}$, then by construction

$$
\mathscr{H}_{0}=\overline{\mathscr{A}_{\text {neutral }} \Omega} \supseteq \mathscr{H}_{0}^{\prime} .
$$

In all models discussed in subsequent sections one may show that, actually,

$$
\mathscr{H}_{0}=\mathscr{H}_{0}^{\prime} .
$$

We therefore shall not distinguish between $\mathscr{H}_{0}$ and $\mathscr{H}_{0}^{\prime}$, anymore.

Theorem 2.3. If the correlation functions $\left\{S_{n, m}\right\}$ defined in (2.13) and (2.14) satisfy hypotheses i), ii) and iii) of Theorem 2.1, and, furthermore, the limits (2.14) vanish, 
then, the Hilbert space $\mathscr{H}$ obtained via reconstruction decomposes into orthogonal sectors, $\mathscr{H}_{q}, q \in \mathscr{Z}$, i.e., for $\hat{F} \in \mathscr{H}_{q}, \hat{G} \in \mathscr{H}_{q}$, with $q \neq q^{\prime}$,

$$
\langle\hat{F}, \hat{G}\rangle=0 \text {. }
$$

Moreover, the sectors $\mathscr{H}_{q}, q \neq 0$, are soliton sectors.

Remark 2.4. If the limits (2.14) do not vanish, then $\mathscr{H}_{q} \subseteq \mathscr{H}_{0}$, for all $q$.

Proof of Theorem 2.3. We first notice that if the limits (2.14) vanish, then, by definition (2.18) of the scalar product, this implies that $\mathscr{H}$ decomposes into orthogonal sectors $\mathscr{H}_{q}$, labelled by the total defect charge, $q$.

Moreover, from (2.16), (2.17), (2.19) it follows that such sectors are invariant under $T, U\left(\right.$ a) and $\mathscr{A}_{0}$. By clustering, the unique translation invariant vector in $\mathscr{H}$ is $\Omega$ which belongs to $\mathscr{H}_{0}$, i.e. for $q \neq 0, \mathscr{H}_{q}$ does not contain any vacuum state.

Remark 2.5. If soliton sectors, $\mathscr{H}_{q}$, exist, then the soliton field operator, $S_{q}(x)$, defined in $\mathscr{H}=\bigoplus_{q} \mathscr{H}_{q}$, maps from the vacuum sector $\mathscr{H}_{0}$ to the soliton sector $\mathscr{H}_{q}$; [see (2.19)]

Theorem 2.3 suggests that there is a general procedure to construct soliton sectors in the Euclidean approach. In fact, such a procedure will be described for kinks, vortices and monopoles in subsequent sections of this paper.

2.3. Classification of Solitons. In this subsection, we define the disorder fields for some lattice field theories and, accordingly, propose a classification of the corresponding solitons. Let us start with the example of the two-dimensional Ising model. We consider joint correlation functions of disorder fields and gaugeinvariant spin fields, such as

$$
\left\langle D(\omega) \sigma_{y_{1}}\left(\prod_{b \in \gamma_{y_{1} y_{2}}} \omega_{b}\right) \sigma_{y_{2}}\right\rangle,
$$

where $\omega=\left\{\omega_{b}\right\}$ is a $\mathbb{Z}_{2}$-valued gauge field, and $\gamma_{y_{1} y_{2}}$ is a path from $y_{1}$ to $y_{2}$. Using gauge invariance, one easily shows that $\left(1.4^{\prime}\right)$ depends on $\omega$ only through its curvature $d \omega$. This property of joint order-disorder correlation functions is not true in general, and the different possible dependences on $\omega$ permit us to classify quantum solitons as follows.

A) Local Solitons. They appear in models in which the expectation value of the disorder field depends on $\omega$ only through $d \omega$.

This property is shared by lattice field theories whose action satisfies the following condition.

\section{Condition $A$ :}

1) $S_{1}$, as defined in Sect. 2.1, is invariant under a group of generalized gauge transformations with values in the discrete, abelian, additive group $\mathscr{Z}$, acting on the highest-rank field, $\phi_{k}$. We suppose that the highest rank is $k$, and by generalized (or hyper-) gauge transformation we mean a gauge transformation whose parameter, $\xi_{k}$, has rank $k$. I.e., we assume that $S_{1}$ is invariant under

$$
\phi_{k}\left(c_{n}\right) \rightarrow \zeta\left(\xi_{k}\left(c_{k}\right)\right) \phi_{k}\left(c_{k}\right), \quad \text { with } \quad \xi_{k}\left(c_{k}\right) \in \mathscr{Z},
$$

where $\zeta$ denotes the representation of $\mathscr{Z}$ in the space of values of $\phi_{k}$. 
2) $S_{0}$ is not invariant under the hyper-gauge transformation (2.22).

We make $S_{0}$ invariant under generalized gauge transformations by a standard procedure: We minimally couple the highest-rank field to an external (hyper-) gauge field $\omega$ of rank $k+1$ with values in $\mathscr{Z}$ and of compact support. We denote by $S_{0}(\omega)$ the action obtained in this way. Hence, by construction, $S_{0}(\omega)$ is invariant under

$$
\left\{\begin{array}{l}
\omega \rightarrow \omega-d \xi_{k} \\
\phi_{k} \rightarrow \zeta\left(\xi_{k}\right) \phi_{k} .
\end{array}\right.
$$

Let us consider a theory with action $S=S_{0}+S_{1}$. Then the disorder field for such a theory is defined by

$$
D(\omega)=e^{-\left[S_{0}(\omega)-S_{0}\right]},
$$

(but see also Remark 3.1).

From the invariance of $S_{0}(\omega)+S_{1}$ under generalized gauge transformations, (2.23), it follows that $\langle D(\omega+d \xi)\rangle=\langle D(\omega)\rangle$. Since the lattice is convex, this implies that the expectation value of $D(\omega)$ depends on $\omega$ only through its curvature $d \omega$. Since $d \omega$ is a field of rank $k+2$ which is closed (i.e. $d(d \omega)=0$ ), it has support in the dual of a set of closed $(d-k-2)$-dimensional surfaces.

From our explicit construction it will be seen that the dimension in which soliton sectors exist is $d=k+2$.

In this particular dimension, the curvature $d \omega$ can be completely characterized by a set of points in the dual lattice, $\left\{x_{i}\right\}_{i=1}^{n}=\operatorname{supp}(d \omega)^{*}$, and by the values of $(d \omega)^{*}$ at these points, i.e. a set of charges $\left\{q_{i} \in \mathscr{Z} \backslash\{0\}\right\}_{i=1}^{n}$. Hence we write

$$
D(\omega) \equiv D\left(x_{1}, q_{1}, \ldots, x_{n}, q_{n}\right) \text {. }
$$

Let $O(\omega . C)$ denote a hyper-gauge invariant, $\omega$-dependent, observable with support in a connected, compact set of cells, $C$. (Some specific choices for concrete models are mentioned in Sect. 3.1.)

Then the correlation functions to which the O-S reconstruction theorem is applied are defined by

$S_{n, m}\left(x_{1}, q_{1}, \ldots, x_{n}, q_{n} ; C_{1}, \ldots, C_{m}\right)=\left\langle D(\omega) O\left(\omega, C_{1}\right) \ldots O\left(\omega, C_{m}\right)\right\rangle$,

with $D(\omega)=D\left(x_{1}, q_{1}, \ldots, x_{n}, q_{n}\right)$, if $\sum_{i=1}^{n} q_{i}=0$ and by (2.14) if $\sum_{i} q_{i} \neq 0$.

The soliton field operator of charge $q, S_{q}(x)$, obtained via reconstruction (see (2.20)) from $\left\{S_{n, m}\right\}$, is local. In particular, for $x=(0, \mathbf{x}), S_{q}(x)$ is defined on the entire Hilbert space and is unitary. Hence, if soliton sectors exist, it is an intertwiner between $\mathscr{H}_{q^{\prime}}$ and $\mathscr{H}_{q^{\prime}+q}$.

In typical examples, a particle structure analysis shows that the soliton field operator $S_{1}(x)$ couples the vacuum to a stable, massive one-particle state, in the phase of the theory, where $\mathscr{H}_{1} \perp \mathscr{H}_{0}$.

In fact, the two-point function of $S_{1}(x), S_{2}(x, 1, y,-1)$, has the long distance behaviour of a two-point function of a massive particle, i.e. an Ornstein-Zernike decay

$$
S_{2}(x, 1, y,-1) \sim \frac{e^{-m|x-y|}}{|x-y|^{d-1 / 2}},
$$

as $\left|x^{0}-y^{0}\right| \rightarrow \infty$, with $m>0$. 
Examples of local soliton sectors are the kink sectors in $2-D \phi^{4}$, in the broken symmetry phase, and the vortex sectors in 3-D Higgs models, in the superconducting phase.

These examples will be discussed in Sects. 3, 4.

B) Stringlike Solitons. String-like solitons are encountered in models where expectation values involving a disorder field, $D(\omega)$, depend on the gauge field $\omega$, rather than merely on $d \omega$. This happens when the part $S_{1}$ of the total action is not invariant under hypergauge transformations, defined in (2.22), i.e.

$$
S_{1}\left(\phi_{k}, \ldots\right) \neq S_{1}\left(\zeta\left(\xi_{k}\right) \phi_{k}, \ldots\right), \quad \xi_{k}\left(c_{k}\right) \in \mathscr{Z} .
$$

The disorder field $D(\omega)$ is defined as in models of class $\mathrm{A}$, but, in order to obtain expectation values satisfying reflection positivity (from which a relativistic quantum theory can be reconstructed), the support of $\omega$ must be chosen appropriately, in particular supp $\omega$ will not be compact, anymore.

Let $\operatorname{supp}(d \omega)^{*}=\left\{x_{1}, \ldots, x_{r}, y_{1} \ldots y_{s}\right\}$, with $x_{i}^{0} \leqq y_{j}^{0}$. Then one may take as the support of $\omega^{*}$ a set of strings, $\gamma_{x_{i}}^{-}$, starting at $x_{i}$ and directed in the negative time direction, $i=1, \ldots, r$, and a set of strings, $\gamma_{y_{j}}^{+}$, starting at $y_{j}$ and directed in the positive time direction, $j=1, \ldots, s$. Let us denote the corresponding disorder field by $D\left(\gamma_{x_{1}}^{-}, q_{1}, \ldots, \gamma_{x_{n}}^{-}, q_{n}, \gamma_{y_{1}}^{+}, q_{r+1}, \ldots, \gamma_{y_{s}}^{+}, q_{r+s}\right)$. With this choice, the support of $\omega$ is infinitely extended in the thermodynamic limit, and, as a consequence, correlation functions involving disorder fields would, in general, vanish, unless they are correctly renormalized. Correlation functions involving disorder fields must therefore be defined as thermodynamic limits of renormalized, finite-volume expectations with appropriate boundary conditions; see Sect. 5 . They are given by

$$
\begin{aligned}
& S_{n=r+s, m}\left(\gamma_{x_{1}}^{-}, q_{1}, \ldots, \gamma_{x_{2}}^{-}, q_{r}, \gamma_{y_{1}}^{+}, q_{r+1}, \ldots, \gamma_{y_{s}}^{+}, q_{r+s} ; C_{1}, \ldots, C_{m}\right) \\
& \equiv \lim _{\Lambda>\mathbb{Z}^{d}} N_{\Lambda}\left(\gamma_{x_{1}}^{-}, q_{1}, \ldots, \gamma_{y_{s}}^{+}, q_{r+s}\right) \\
& \quad\left\langle D\left(\gamma_{x_{1}}^{-}, q_{1}, \ldots, \gamma_{y_{s}}^{+}, q_{r+s}\right) O\left(C_{1}\right), \ldots, O\left(C_{m}\right)\right\rangle_{\Lambda},
\end{aligned}
$$

if $\sum_{i=1}^{r+s} q_{i}=0$. Here $N_{\Lambda}$ is a suitable normalization factor which ensures that the limit exists and is non-zero and $x_{i}, y_{i}, \omega$ are defined as above. Correlation functions of non-zero total charge are defined by removing a charge to infinity, as in (2.14). The soliton field operator $S_{q}\left(\gamma_{x}\right)$ reconstructed from such correlation functions is a lattice version of a field localized in a cone, as studied by Buchholz and Fredenhagen in [6].

For a variety of lattice theories, one can show that, in the phase where stringlike soliton sectors exist, the two-point function of $S_{1}\left(\gamma_{x}\right)$ decays like

$$
\left\langle S_{1}\left(\gamma_{x}\right) \Omega, S_{1}\left(\gamma_{y}\right) \Omega\right\rangle \equiv S_{2}\left(\gamma_{x}^{-},-1, \gamma_{y}^{+}, 1\right) \sim \frac{e^{-m|x-y|}}{|x-y|^{(d-1) / 2}}
$$

as $\left|x^{0}-y^{0}\right| \rightarrow \infty$, with $m>0$. Hence, the soliton field operator $S_{1}\left(\gamma_{x}\right)$, couples the vacuum to a stable massive one-particle state.

Examples of string-like soliton sectors are found in 3-D $\mathbb{Z}_{N}$-Higgs models, where they are dual to the charged sectors constructed in [13]. They thus correspond to the 
$\mathbb{Z}_{N}$ vortices. Another interesting example is a Higgs model in three dimensions, in the superconducting phase, weakly coupled to fractionally charged fermions. Such three space-time dimensional theories may exhibit particles with intermediate spin and statistics, called "anyons" [14]. They might be relevant in a theory of the fractional quantum Hall effect [15]. For a rigorous analysis of such theories, see also [16]. The two examples mentioned above and a 2-D example will be discussed in Sect. 5.

C) Monopoles. Consider a theory (such as the compact $U(1)_{4}$-gauge model) which can be expressed as a gas of current loops, with the property that every current loop carries a magnetic flux indexed by the elements of a discrete, abelian group $\mathscr{Z}$. In such a theory, a disorder field is constructed by opening up a current loop, i.e. introducing an open-ended current line, $\omega$. Since magnetic flux is conserved, a disorder field must always be accompanied by a magnetic field, $B$, with sources at the end points of the open current line $\omega$. The classical field $B$ can be chosen in many different ways, subject to the constraint that its sources coincide with the endpoints of $\omega$ and that the total magnetic flux is conserved. The corresponding disorder field is denoted by $D(\omega, B)$, and the sectors are called monopole sectors. An example which is under control concerns the monopole sectors in the $U(1)_{4}$-gauge theory, in the Q.E.D. phase.

Typical choices for $B$ are Coulomb fields in a fixed time plane, spreading out symmetrically from the endpoints of $\omega$, or fields supported in cones with apex in an endpoint of $\omega$.

The disorder field is defined as follows: Let $\omega$ be a third-rank, $\mathbb{Z}$-valued field with $(d \omega)^{*}$ supported in $\left\{x_{i}\right\}_{i=1}^{n}$, and $(d \omega)^{*}\left(x_{i}\right)=q_{i} \in \mathbb{Z}, i=1, \ldots, n$. Let $B\left(x_{i}, q_{i}\right)$ be a magnetic field, as envisaged above, with $\delta B\left(x_{i}, q_{i}\right)=q_{i} \delta_{x_{i}}$. We set

$$
B=\sum_{i=1}^{n} B\left(x_{i}, q_{i}\right)
$$

and let $\theta$ denote the $U(1)$-valued gauge field of the model. Then

$$
\langle D(\omega, B)\rangle=\frac{Z(\omega, B)}{Z},
$$

where $Z(\omega, B)$ is the partition function obtained from the partition function, $Z$, of the $U(1)_{4}$-gauge model by making the following substitution in the usual Wilson- or Villain action:

$$
d \theta \rightarrow d \theta+2 \pi \delta \Delta^{-1}(B-\omega) .
$$

In the representation of $\langle D(\omega, B)\rangle$ in terms of magnetic currents, open currents ending at $\left\{x_{i}\right\}$ appear, accompanied by magnetic fields $B\left(x_{i}, q_{i}\right)$. This representation is discussed in more detail in Sect. 6.

Using the periodicity of the action in $\theta$ and the Hodge decomposition

$$
\xi=\delta \Delta^{-1} d \xi+d \Delta^{-1} \delta \xi,
$$

one easily sees that $\langle D(\omega, B)\rangle$ is invariant under the gauge transformation

$$
\omega \rightarrow \omega+d \xi
$$

where $\xi$ is a rank-2, $\mathbb{Z}$-valued field; i.e. $\langle D(\omega, B)\rangle$ depends only on $d \omega$ and $B$. 
Since $\omega^{*}$ can be interpreted as a set of Dirac strings attached to the monopoles, invariance under (2.31) corresponds to the invisibility of the Dirac strings in a theory without dynamical electric monopoles.

Invariance under (2.31) holds also if we consider expectation values involving periodic functions of $d \theta-2 \pi \delta \Delta^{-1} \omega$, such as

$$
\begin{aligned}
& O(\omega, p)=e^{i\left(d \theta-2 \pi \delta \Delta^{-1} \omega\right)_{p}}, \quad \text { or } \\
& O(\omega, S)=\prod_{b \in C} e^{i \theta_{b}} \prod_{p \in S: \partial S=C} e^{-i 2 \pi\left(\delta \Delta^{-1} \omega\right)_{p}} .
\end{aligned}
$$

The correlation functions to which the reconstruction theorem applies are given by $S_{n, m}\left(x_{1}, q_{1}, \ldots, x_{n}, q_{n} ; S_{1}, \ldots, S_{m}\right)=\left\langle D(\omega, B) O\left(\omega, S_{1}\right), \ldots, O\left(\omega, S_{m}\right)\right\rangle$,

for $\sum_{i} q_{i}=0$, where we have chosen a fixed shape, $B_{0}$, for all magnetic fields, i.e. $B(x, q)=q\left(B_{0}\right)_{x}$, and we have omitted any reference to $B_{0}$ in the correlation functions. The monopole field operator obtained via reconstruction, $S_{q}\left(x, B_{0}\right)$, is non-local. It is localized on $\operatorname{supp} B(x, q)$, i.e. at best on space-like cones with strictly positive opening angle. The classical $B$-field appears to introduce corrections to the Ornstein-Zernike decay of the monopole two-point function $S_{2}(x, 1, y,-1)$, exhibiting the infraparticle nature of the monopole. More precisely, we expect that

$$
S_{2}(x, 1, y,-1) \sim \frac{e^{-m|x-y|}}{|x-y|^{[(d-1) / 2]+\gamma}}
$$

as $\left|x^{0}-y^{0}\right| \nearrow \infty$, with $m>0, \gamma>0$.

Some comments on the monopole sectors in the $U(1)_{4}$ gauge theory are made in Sect. 6. See also $[1,17]$.

\section{Local Solitons}

3.1. Reflection Positivity of Correlation Functions. In this section, we consider lattice field theories satisfying condition $A$ of Sect. 2.3 which exhibit local solitons. We discuss some explicit examples and prove reflection positivity of the correlation functions defined in Sect. 2.3.

Condition $A$ is satisfied in two classes of theories:

I. Scalar or fermionic theories in two space-time dimensions with a discrete global symmetry group, $\mathscr{Z}$.

II. Gauge theories in three space-time dimensions with a matter field action invariant under a subgroup, $\mathscr{Z}$, of the center of the gauge group.

We consider the following examples.

A1) $\phi_{2}^{4}$ Model. In this model the scalar field is real, and the potential is given by

$$
V\left(\phi_{x}\right)=\lambda\left(\phi_{x}^{2}-1\right)^{2} .
$$

To obtain a unique vacuum one should choose + or - boundary conditions.

The "hypergauge" transformation for this model is given by

$$
\phi_{x} \rightarrow e^{i \pi z_{x}} \phi_{x} \quad z_{x} \in \mathbb{Z}_{2} \simeq\{0,1\} .
$$


A2) Massless Yukawa, Model. This model is obtained by adding a fermion field to the $\phi^{4}$ model and setting

$$
S_{1}(\bar{\psi}, \psi, \phi)=\lambda \sum_{x} \bar{\psi}_{x} \phi_{x} \psi_{x}
$$

In the kinetic term of the fermionic action we use naive lattice fermions (see (2.9)). This choice does not give the right continuum limit, but, as far as lattice theory is concerned, it is perfectly consistent.

This model possesses two "hypergauge symmetries":

$$
\begin{aligned}
\phi_{x} & \rightarrow e^{i \pi z_{x}} \phi_{x} \\
\phi_{x} & \rightarrow e^{i \pi z_{x}} \phi_{x} \quad \psi_{x} \rightarrow e^{i \frac{\pi}{2} \gamma_{5} z_{x}} \psi_{x} \rightarrow e^{-i \frac{\pi}{2} \gamma_{5} z_{x}} \psi_{x},
\end{aligned}
$$

with $z_{x} \in \mathbb{Z}_{2} \simeq\{0,1\}$.

A3) Non-Compact Abelian Higgs $s_{3}$ Model. In this model, the gauge field, A, is real-valued, and the Higgs field, $\phi$, is complex.

The $S_{1}$-term in the action is given by

$$
S_{1}(A, \phi)=\frac{\beta_{H}}{2} \sum_{\langle x y\rangle}\left|\phi_{y}-e^{i A\langle x y\rangle} \phi_{x}\right|^{2}+g \sum_{x}\left(\left|\phi_{x}\right|^{2}-1\right)^{2} .
$$

The "hyper-gauge transformation" is given by

$$
A_{\langle x y\rangle} \rightarrow A_{\langle x y\rangle}+2 \pi z_{\langle x y\rangle}, \quad z_{\langle x y\rangle} \in \mathbb{Z} .
$$

A4) $S U(N)-$ Higgs $_{3}$ Model. With gauge fields in the fundamental representation and Higgs fields in the adjoint representation, the "hyper-gauge transformation" is given by

$$
g_{\langle x y\rangle} \rightarrow e^{i \frac{2 \pi}{N} z_{\langle x y\rangle}} g_{\langle x y\rangle}, \quad z_{\langle x y\rangle} \in \mathbb{Z}_{N} \simeq\{0, \ldots, N-1\} .
$$

A5) Rank-k Stückelberg Models. These are higher-rank gauge theories with matter fields. The gauge field, $A$, has rank $k>1$ and is real-valued, the matter field, $B$, has rank $k-1$ and is $U(1)$-valued.

The action is given by $S=S_{0}+S_{1}+S_{\text {fix }}$, with

$$
S_{0}\left(A_{k}\right)=\frac{\beta_{G}}{2} \sum_{c_{k+1}}\left[d A\left(c_{k+1}\right)\right]^{2}
$$

and

$$
S_{1}(A, B)=\beta_{H} \sum_{c_{k}} \cos \left[A\left(c_{k}\right)+d B\left(c_{k}\right)\right]
$$

and some gauge fixing term, $S_{\text {fix }}(A)$, is added. For $k=0$, setting $B_{-1} \equiv 0$, we obtain the sine-Gordon model, and, for $k=1$, the usual Stückelberg model. The hypergauge transformation is given by

$$
A_{k}\left(c_{k}\right) \rightarrow A_{k}\left(c_{k}\right)+2 \pi z\left(c_{k}\right), \quad z\left(c_{k}\right) \in \mathbb{Z} .
$$

We now turn to the problem of constructing joint order-disorder correlation functions for these models. 
As mentioned in Sect. 2.3, the disorder field is obtained by replacing $S_{0}$ by $S_{0}(\omega)$. This corresponds to the following substitutions in $S_{0}$ : In $2-D$ scalar theories,

$$
\phi_{y}-\phi_{x} \rightarrow \phi_{y}-\zeta_{H}\left(\omega_{\langle x y\rangle}\right) \phi_{x}
$$

and in $2-D$ Fermionic theories

$$
\bar{\psi}_{x} \Gamma_{\langle x y\rangle} \psi_{y} \rightarrow \bar{\psi}_{x} \Gamma_{\langle x y\rangle} \zeta_{F}\left(\omega_{\langle x y\rangle}\right) \psi_{y}
$$

In $3-D$ gauge theories,

$$
\chi\left(g_{\partial p}\right) \rightarrow \chi\left(g_{\partial p} \zeta_{G}\left(\omega_{p}\right)\right)
$$

or

$$
(d A)\left(c_{k+1}\right) \rightarrow d A\left(c_{k+1}\right)+2 \pi \omega\left(c_{k+1}\right) .
$$

Here $\zeta_{H}, \zeta_{F}, \zeta_{G}$ are the representations of $\mathscr{Z}$ on $V_{H}, V_{F}, G$, respectively.

By hypergauge invariance, expectation values of products of disorder fields and hypergauge-invariant functions of the basic fields depend on $\omega$ only through $d \omega$. Since the basic observables are not always hypergauge invariant, we have to modify them, by explicitly introducing a dependence on the external (hyper) gauge field $\omega$. For example, in scalar and Fermion theories, we shall work with the observables

$$
O\left(\omega, C_{x y}\right)=\bar{\phi}_{x} \prod_{b \in C_{x y}} \zeta_{H}\left(\omega_{b}\right) \phi_{y}, \quad \text { or } \quad=\bar{\psi}_{x} \prod_{b \in C_{x y}} \zeta_{F}\left(\omega_{b}\right) \psi_{y},
$$

where $C_{x y}$ is a curve from $x$ to $y$, instead of using $\left\{\phi_{x}, \bar{\psi}_{x}, \psi_{x}\right\}$.

For gauge theories with matter fields, the standard observables, the Wilson loop, $\chi_{H}\left(g_{C}\right)$, and the string variables $\bar{\phi}_{x} \prod_{b \in C_{x y}} U_{H}\left(g_{b}\right) \phi_{y}$ are already hypergaugeinvariant, provided $\chi_{H}$ is a character of $G / \mathscr{Z}^{x}$.

If we want to use a Wilson loop with a character $\chi$ which is not $\mathscr{Z}$-invariant then we have to use the modified observable

$$
O(\omega, S)=\chi\left(g_{C} \prod_{p \in S: \partial S=C} \zeta_{G}\left(\omega_{p}\right)\right) .
$$

If expectation values of functions of the basic fields, $O_{i}$, which are not hypergauge-invariant are considered, they depend on $\omega$ only through the homology class of supp $\omega$ in $\mathbb{Z}_{1 / 2}^{d} \backslash \operatorname{supp} O_{i}$. For example, in model A4), the transformation $\omega \rightarrow \omega+d \xi$, with $\xi$ a $\mathbb{Z}_{N}$-valued field of rank 1 , induces a change in $\left\langle D(\omega) \chi\left(g_{C}\right)\right\rangle$ only if $\operatorname{supp}(d \xi) *$ links $C$, and

$$
\left\langle D(\omega+d \xi) \chi\left(g_{C}\right)\right\rangle=e^{i \frac{2 \pi}{N} n(\xi, C)}\left\langle D(\omega) \chi\left(g_{C}\right)\right\rangle,
$$

where $n(\xi, C)$ is the linking number of supp $(d \xi)^{*}$ and $C$. However, to avoid all these homological factors, we use only correlation functions involving hypergaugeinvariant order fields.

We now propose to prove O-S positivity of the correlation functions $\left\{S_{n, m}\right\}$, defined in (2.25), in dimension $d=k+2$. All the models we have introduced above have an $\mathrm{O}-\mathrm{S}$ positive vacuum functional. Hence our claim concerning O-S positivity of sequences of correlation functions $\left\{S_{n, m}\right\}$ is proved if there exists a field 
$D_{+}\left(x_{1}, q_{1}, \ldots, x_{n}, q_{n}\right)$ in $\mathscr{F}_{+}$(i.e. depending only on the field variables located on the positive time lattice, $\left.\mathbb{Z}_{+}^{d}\right)$, such that for $x_{i}, y_{j} \in \mathbb{Z}_{+}^{d}$ :

$$
\begin{aligned}
& \left\langle D\left(x_{1}, q_{1}, \ldots, x_{n}, q_{n}, r y_{1},-q_{1}^{\prime}, \ldots, r y_{m}-q_{m}^{\prime}\right) \cdot \prod_{i} O\left(\omega, C_{i}\right) \theta\left[\prod_{j} O\left(\omega, C_{j}\right)\right]\right\rangle \\
& =\left\langle D_{+}\left(x_{1}, q_{1}, \ldots, x_{n}, q_{n}\right) \prod_{i} O\left(\omega, C_{i}\right) \theta\left[D_{+}\left(y_{1}, q_{1}^{\prime}, \ldots, y_{m}, q_{m}^{\prime}\right) \cdot \prod_{j} O\left(\omega, C_{j}\right)\right]\right\rangle .
\end{aligned}
$$

This, in turn, follows from the arbitrariness in the choice of $\omega$, with $d \omega$ fixed, that follows from hypergauge invariance.

Let us suppose, for simplicity, that $\sum_{i} q_{i}=q=\sum_{j} q_{j}^{\prime}$, then

$$
\begin{aligned}
& \left\langle D\left(x_{1}, q_{1}, \ldots, x_{n}, q_{n}, r y_{1},-q_{1}^{\prime}, \ldots, r y_{m},-q_{m}^{\prime}\right)(\ldots)\right\rangle \\
& \quad=\left\langle D\left(x_{1}, q_{1}, \ldots, x_{n}, q_{n}, 0,-q\right) D\left(r y_{1},-q_{1}^{\prime}, \ldots, r y_{m},-q_{m}^{\prime}, 0, q\right)(\ldots)\right\rangle .
\end{aligned}
$$

Moreover, we can choose the support of $\omega^{*}$ to be a set of paths starting at $x_{i} \in \mathbb{Z}_{+}^{d}\left(r y_{j} \in \mathbb{Z}_{-}^{d} \equiv r\left(\mathbb{Z}_{+}^{d}\right)\right.$, respectively) and ending at 0 completely lying in the strictly positive (negative) time lattice and such that the path starting at $x_{i}$ is the reflection of the path starting at $r x_{i}$.

With this choice

$$
\begin{aligned}
& D_{+}\left(y_{1}, q_{1}^{\prime}, \ldots, y_{n}, q_{n}^{\prime}\right) \equiv D\left(y_{1}, q_{1}^{\prime}, \ldots, y_{n}, q_{n}^{\prime}, 0,-q^{\prime}\right) \\
& \quad=\theta D\left(r y_{1},-q_{1}^{\prime}, \ldots, r y_{n},-q_{n}^{\prime}, 0, q^{\prime}\right)=\theta D_{+}\left(\left(r y_{1},-q_{1}^{\prime}, \ldots, r y_{n},-q_{n}^{\prime}\right) .\right.
\end{aligned}
$$

For the two-point function, our proof is illustrated in Fig. 0.

Since lattice translation invariance and reflection positivity hold for $\left\{S_{n, m}\right\}_{n, m=0}^{\infty}$, we can apply, to these sequences, the reconstruction theorem and obtain a Hilbert space of states $\mathscr{H}$, the vacuum $\Omega$, the transfer matrix $T$, the unitaries $U_{\mu}$, the order field operators $A(C)$ and the soliton field operators $S_{q}(x)$.

If we prove clustering and show that the limits considered in (2.14) vanish, then, by Theorem 2.3, we obtain soliton sectors $\mathscr{H}_{q}$, labelled by a charge $q \neq 0$ with values in $\mathscr{Z}$. To prove clustering, we first rewrite the joint order-disorder correlation functions in terms of defects (Sect. 3.3) and then use a cluster expansion (Sect. 3.4).

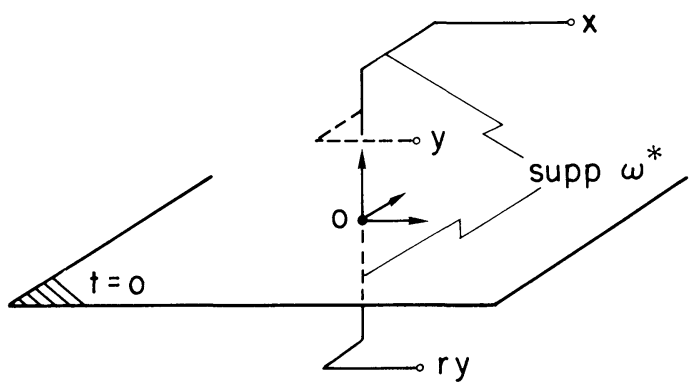

Fig. 0 


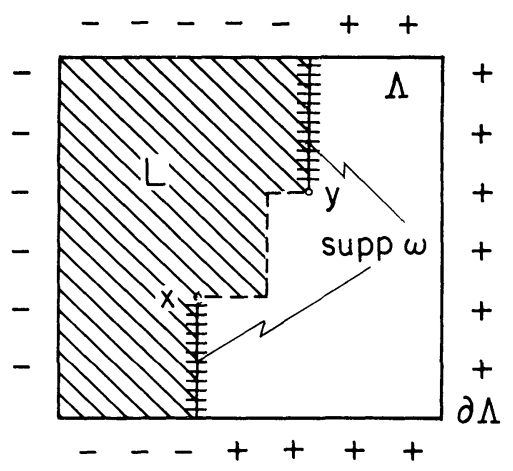

Fig. 1

Remark. We should mention that there is an alternative construction of soliton Green functions similar to the one used in models with non-local solitons and more akin to the Hamiltonian approach to soliton quantization. In the example of the kink propagator in $\lambda \phi_{2}^{4}$-theory this modified construction involves imposing +- boundary conditions (see Fig. 1 ) and choosing the support of the external gauge field $\omega$ to consist of two strings attached to the positions, $x$ and $y$, of the kinks and extending to the boundary of the system, as sketched in Fig. 1. This construction of the kink propagator is related to the previous one by a global $\mathbb{Z}_{2}$ gauge transformation; $\phi(x) \rightarrow-\phi(x)$, for $x \in L$, with support on the half space $L$. It therefore yields an identical kink propagator. Clearly, it extends to arbitrary soliton Green functions, reproducing the same results as those obtained before.

Similar remarks apply to the other examples of theories with local solitons discussed above, e.g. the vortices in three-dimensional Higgs models.

3.2. Defect Representation of Soliton Euclidean Green Functions. A defect representation of the joint order-disorder correlation functions has several applications:

It provides an intuitive idea of what the disorder field is and how it is related to solitons; it suggests the basic structure of the cluster expansion needed to prove the clustering of the correlation functions and the vanishing of the limits in (2.14); and it also plays a central role in the analysis of the particle structure of soliton sectors. To simplify our notations, we consider only disorder correlation functions, but hypergauge-invariant observables can be included in a straightforward way.

Let us start by discussing the partition function, $Z$, of the $\phi_{2}^{4}$ model. The simplest way to reveal the defects is to rewrite $Z$ in terms of the "hypergaugeinvariant" field $\varrho_{x}=\left|\phi_{x}\right|$ and the $\mathbb{Z}_{2}$-valued "spin field", $\sigma_{x}$, defined by

$$
\phi_{x}=e^{l \pi \sigma_{x}} \varrho_{x}=\operatorname{sign} \phi_{x}\left|\phi_{x}\right|
$$

The Peierls contours for the spin field $\sigma_{x}$, or, in other words, the connected components of $\operatorname{supp}(d \sigma)^{*}$, are the defects of the model. 
This way of exhibiting the defects can be generalized to discuss all hypergaugeinvariant order-disorder correlation functions of the models satisfying condition $A$ of Sect. 2.3. Consider e.g. the expectation value

$$
\langle D(\omega)\rangle=\frac{Z(\omega)}{Z} .
$$

Rewrite $Z(\omega)$ in terms of a set of fields which represent orbits of the basic fields under the hypergauge transformation (2.22), such as $\varrho_{x}$ in $\phi^{4}$, and a $\mathscr{Z}$-valued field, $\sigma$, of rank $k$ parametrizing the orbits.

By the hypergauge invariance of $S_{1}, \sigma$ can only appear in $S_{0}(\omega)$, and from the hypergauge invariance of $S_{0}(\omega)$ it follows that $\sigma$ appears only in the hypergaugeinvariant expression

$$
v=d \sigma+\omega .
$$

Example. In the non-compact abelian Higgs model, A3), we decompose the gauge field as

$$
A_{\langle x y\rangle}=\theta_{\langle x y\rangle}+2 \pi \sigma_{\langle x y\rangle}, \theta_{\langle x y\rangle} \in(-\pi, \pi), \sigma_{\langle x y\rangle} \in \mathbb{Z},
$$

and take as our orbit fields $(\theta, \phi)$, where $\phi$ is the Higgs field on which the hypergauge transformations act trivially.

The action of the model coupled to $\omega$ can be rewritten as

$$
S_{0}(\omega)+S_{1}=\frac{\beta_{G}}{2} \sum_{p}\left(d \theta_{p}+2 \pi v_{p}\right)^{2}+S_{1}(\theta, \phi),
$$

using the periodicity of $S_{1}$ in $A$. Hence it depends only on the orbit fields $(\theta, \phi)$ and the field $v$.

We now turn to the general case. Define $Z(v)$ to be the function obtained by integrating $e^{-\left[S_{0}(\omega)+S_{1}\right]}$ over all orbit fields, for a fixed configuration, $v$, of defects. Then we get

$$
\langle D(\omega)\rangle=\frac{Z(\omega)}{Z}=\frac{\sum_{v: d v=d \omega} Z(v)}{\sum_{v: d v=0} Z(v)} .
$$

The object $v$ in the denominator is a $\mathscr{Z}$-valued field of rank $k+1$ and is closed. The support is therefore dual to a set of closed $d-k-1$ dimensional surfaces, i.e. a set of loops when $d=k+2$.

These loops are just the supports of the $\mathscr{Z}$ defects generalizing the notion of Peierls contours in the Ising or the $\phi^{4}$-model. For the non-compact abelian Higgs model, they are the Abrikosov vortex loops.

The $v$ in the numerator of (3.2) satisfies $d v=d \omega$. Hence it has support in the dual of a set of $d-k-1$ dimensional surfaces, whose boundary is given by $\operatorname{supp}(d \omega)^{*}$, a set of $d-k-2$ dimensional surfaces (see Fig. 2).

Therefore, for $d=k+2$, Eq. (3.2) expresses $Z(\omega)$ as the partition function of a gas of line defects labelled by the elements of $\mathscr{Z}$. If $\omega \neq 0$, then $Z(\omega)$ is a sum over configurations of open line defects whose boundary is given by $\operatorname{supp}(d \omega)^{*}$, and of closed defects. 
a)

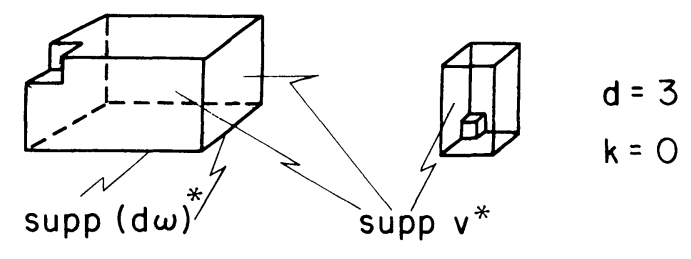

b)
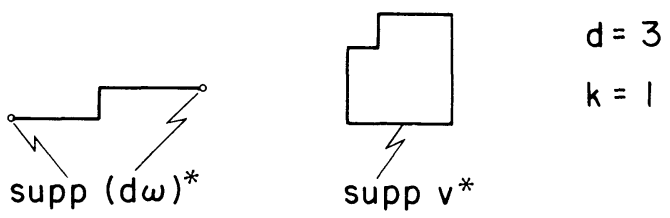

Fig. 2. Configuration of $v$ occurring in the numerator of (3.2)

Hence Eq. (3.2) is a generalization of (1.3). In a particle picture, one can interpret the closed defects as Euclidean worldlines of pairs of virtual solitons.

An open line defect corresponds to the worldline of a soliton created at one end of the line and annihilated at the other one. From this point of view, it is clear that solitons in models satisfying condition $A$ exist when the dimension is $d=k+2$. For this value of $d$ the defects are line defects.

Remark 3.1. It is possible to use a definition of the disorder field slightly different from Eq. (2.20), involving the $\mathscr{Z}$-valued field $\sigma$ defined above. Let $\{O\}$ denote the set of orbit fields considered at the beginning of the section.

The disorder field is then defined to be an operator $D_{\mathrm{op}}(\omega)$ acting on function $F(O, d \sigma)$ of $O$ and $d \sigma$ by

$$
D_{\mathrm{op}}(\omega) F(O, d \sigma)=F(O, d \sigma+\omega) \equiv F(\omega) .
$$

Hence, denoting by $d O$ the measure over the orbit fields,

$$
\begin{aligned}
& \sum_{\sigma} \int d O D_{\mathrm{op}}(\omega) e^{-\left[S_{0}(O, d \sigma)+S_{1}(O)\right]} F(O, d \sigma) \\
& \quad=\sum_{\sigma} \int d O e^{-\left[S_{0}(O, d \sigma+\omega)+S_{1}(o)\right]} F(O, d \sigma+\omega)=\langle D(\omega) F(\omega)\rangle .
\end{aligned}
$$

3.3. Clustering. For models involving only a $\mathscr{Z}$-valued field, the proof of clustering in the low-temperature region follows immediately from Eq. (3.2) by a standard low-temperature (L.T.) cluster expansion.

In the 2-D Ising model, at large $\beta$, one can rewrite (3.2) as, (see e.g. [12]):

$$
\langle D(\omega)\rangle=\sum_{v^{\omega}} z\left(v^{\omega}\right),
$$

where $v^{\omega}$ denotes a configuration of the $v$-field such that every connected component of $\operatorname{supp}\left(v^{\omega}\right)^{*}$ has non-vanishing intersection with $\operatorname{supp}(d \omega)^{*}$ and $d v^{\omega}=d \omega$. Hence, in (3.4), supp $\left(v^{\omega}\right)^{*}$ is given by a set of fluctuating random lines 
joining the points of $\operatorname{supp}(d \omega)^{*} . z\left(v^{\omega}\right)$ is a statistical weight explicitly given by (see [12])

$$
z\left(v^{\omega}\right)=e^{-2 \beta\left|v^{\omega}\right|} \sum_{\mathbf{v}} \prod_{v \in \mathbf{v}} e^{-2 \beta|v|} a_{T}\left(\mathbf{v}, v^{\omega}\right),
$$

where $v$ denotes a configuration of the $v$-field with $\operatorname{supp} v^{*}$ connected and $d v=0 ; \mathbf{v}$ denotes a collection of $v$ 's where every $v$ can occur an arbitrary number of times, and $a_{T}\left(\mathbf{v}, v^{\omega}\right)$ is a combinational factor which vanishes, unless every connected component of $\operatorname{supp}(\mathbf{v})^{*}$ touches $\operatorname{supp}\left(v^{(\omega)}\right)^{*}$. Finally $|v|=\operatorname{card}(\operatorname{supp} v)$. One easily establishes an upper bound

$$
\left|z\left(v^{\omega}\right)\right| \leqq e^{-\left[2 \beta-O\left(e^{-\beta}\right)\right]\left|v^{\omega}\right|}
$$

from which clustering and the vanishing of the limit in (2.14) follow.

If, in a lattice model, continous fields are present as well, one can still derive from (3.2) a representation of disorder correlation functions similar to (3.4). In fact, every configuration of defects appearing in the numerator of (3.2) contains a component $v^{\omega}$ with $d v^{\omega}=d \omega$ as above, and a set of closed components, $v_{i}$, with $d v_{i}=0$ and $\operatorname{supp} v_{i}^{*}$ connected. If we resum (3.2) over the closed defects, $v_{i}$, then we obtain a representation (3.4), where $z\left(v^{\omega}\right)$ is the statistical weight of the defect $v^{\omega}$ interacting with a gas of closed defects. In order to prove clustering, however, we now need to combine a low-temperature expansion for the $v$-field, as before, with a high-temperature expansion for the other fields (i.e. the orbit fields of Sect. 3.2).

The combined expansion will be called "combined low- and high-temperature (C.L.H.T.) expansion." Generally speaking, these expansions tend to converge in the phase in which the gas of defects is dilute, i.e. when $d=2$, in the phase where the $\mathscr{Z}$-symmetry is spontaneously broken, and when $d \geqq 3$ in the superconducting phase.

The upshot of the C.L.H.T. expansion is an estimate like

$$
\left|z\left(v^{\omega}\right)\right| \leqq e^{-c\left|v^{\omega}\right|} .
$$

Again, an estimate like (3.6) yields clustering of correlations and proves vanishing of the limits (2.14), by standard arguments.

In our examples, those expansions converge in A1), A2), $(d=2)$, for $\beta_{H}, \lambda$ large, $\beta_{F}$ small, with $c=O\left(\beta_{H}\right)$; in A3), A4), $(d=3)$, for $\beta_{G}, \beta_{H}, \lambda$ large, with $c=O\left(\beta_{G}\right)$; in A5), $(d=k+2)$, for $\beta_{G}, \beta_{H}$ large, with $c=O\left(\beta_{G}\right)$. Details will appear in [18].

Since the C.H.L.T. expansion is not yet completely standard, (but see e.g. [12, Quasi-theorem 3.20], and [19]), we give an outline of how it works for disordercorrelation-functions in Appendix 1.

Clearly, the limits (2.4) do not vanish in the "high-temperature region" of parameter space, where the defects, $v^{*}$, condense, (i.e. the confinement region for gauge theories). As a consequence soliton sectors do not exist.

This discussion is summarized, pictorially, in Fig. 3. The shaded regions correspond to the phases in which soliton sectors exist.

Remark 3.2. If the correlation functions $\left\{S_{n, m}\right\}_{n, m=0}^{\infty}$ admit a Euclidean-invariant continuum limit, as one expects for all models discussed in this section, then a 


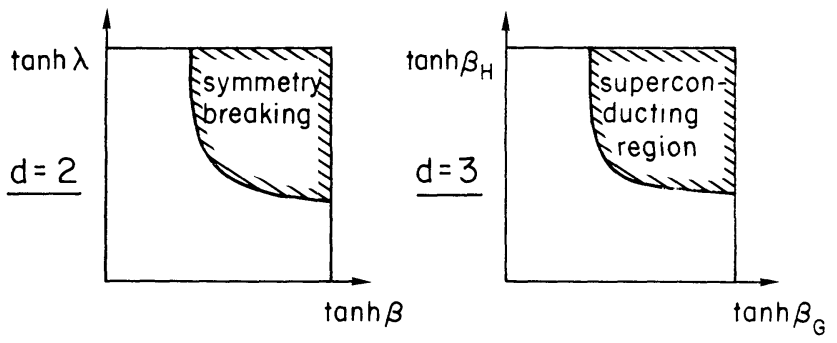

Fig. 3

unitary representation of the full Poincaré group satisfying the spectral condition can be constructed [11].

A fully relativistic Q.F.T. with soliton sectors is then obtained by OsterwalderSchrader reconstruction.

Remark 3.3. The soliton sector constructed in the 2-D $\phi^{4}$ models for + b.c., is the so-called kink sector. If we consider $\phi_{2}^{4}$ with - b.c. an anti-kink sector is obtained. Both sectors have been constructed in the continuum in a somewhat abstract fashion in $[8,9]$. The soliton operator, $S(0)$, can be considered to be a lattice version of the soliton intertwiner introduced in $[8,9]$.

There are two soliton sectors in the massless (Yukawa) model, with + b.c. for $\phi$, corresponding to the two symmetry transformations

$$
\psi_{x} \rightarrow e^{i \frac{\pi}{2} \gamma_{5}} \psi_{x}, \quad \psi_{x} \rightarrow e^{-i \frac{\pi}{2} \gamma_{5}} \psi_{x} .
$$

The solitons have fractional fermion numbers, $1 / 2$ and $-1 / 2$, respectively. (For a heuristic discussion based on the W.K.B. approximation see e.g. [20]. A rigorous analysis appears in [16].)

The soliton sectors of the 2- $d$ sine-Gordon model ( $\equiv$ rank-O Stückelberg model), too, have been constructed in the continuum in $[9,21]$.

\section{Particle Structure in Soliton Sectors}

In this section, we discuss the particle structure on local soliton sectors, using the excitation analysis of [4].

We consider the models discussed in Sect. 3.1, (A1-5). Our goal is to show that, in the phase of a model where the soliton two-point function does not have longrange order, the soliton field operator with lowest charge, $S_{1}(x)$, couples the vacuum to a stable, massive one-particle state. This supports the general idea that the soliton is a massive particle.

4.1. General Remarks on Particle Structure. We define the mass operator of a lattice field theory by [4]:

$$
M=-\ln \left(T \uparrow \mathscr{H}^{(0)}\right)
$$


where $\mathscr{H}^{(0)}$ denotes the subspace of zero total momentum in the direct integral representation of $\mathscr{H}$, i.e. $\psi \in \mathscr{H}^{(0)}$ iff $U(\mathbf{a}) \psi=\psi$.

Applying the spectral representation to $T$ and $U$, we obtain, for every operator $A \in \mathscr{A}, t \geqq 0$ (see Sect. 2.2):

$$
\begin{aligned}
\langle A \Omega ; U(\mathbf{x}) T(t) A \Omega\rangle & \equiv\langle A \Omega, U(\mathbf{x}) T(t) A \Omega\rangle-\langle A \Omega, \Omega\rangle\langle\Omega, A \Omega\rangle \\
& =\int d \varrho_{A}(\lambda, \mathbf{k}) \lambda^{t} e^{i \mathbf{k} \cdot \mathbf{x}},
\end{aligned}
$$

where $d \varrho_{A}(\lambda, \mathbf{k})$ is a finite positive measure with support in $[\alpha, 1] \times[-\pi, \pi]^{d-1}$, and $\alpha \geqq-1$. In particular, $\alpha=0$ if $T \geqq 0$, which holds in our models without fermions.

By summing the lefthand side of (4.2) over $\mathbf{x} \in \mathbb{Z}^{d-1}$, we obtain

$$
\sum_{\mathbf{x}}\langle A \Omega ; U(\mathbf{x}) T(t) A \Omega\rangle=\int d \varrho_{A}(\lambda, \mathbf{0}) \lambda^{t} .
$$

Comparison with (4.1) gives

$$
\operatorname{spec} e^{-M} \supseteq \operatorname{supp} d \varrho_{A}(\lambda, \mathbf{0}) .
$$

Since $A \Omega$ is dense in $\mathscr{H}$ we obtain

$$
\operatorname{spec} e^{-M}=\bigcup_{A \in \mathscr{A}}\left\{\operatorname{supp} d \varrho_{A}(\lambda, \mathbf{0})\right\},
$$

where the bar denotes the closure in $\mathbb{R}$.

Using (4.3), (4.4) one notices that $A$ couples the vacuum $\Omega$ to a stable oneparticle state iff

$$
\sum_{\mathbf{x}}\langle A \Omega ; U(\mathbf{x}) T(t) A \Omega\rangle \underset{t>\infty}{\sim} e^{-m(A) t}\left(1+\text { const } e^{-\mu(A) t}\right),
$$

with $m(A), \mu(A)>0$.

If $\mu(A)=0$, one can still show that a decay

$$
\langle A \Omega ; T(t) U(\mathbf{x}) A \Omega\rangle \underset{t \nearrow \infty}{\sim} \frac{e^{-m(A) t}}{t^{(d-1) / 2}}
$$

with $m(A)>0$, is equivalent to the statement that $A$ couples $\Omega$ to a stable oneparticle state. This decay law is called Ornstein-Zernike decay.

More generally, if

$$
\langle A \Omega ; T(t) U(\mathbf{x}) A \Omega\rangle \underset{t \succ_{\infty}}{\sim} \frac{e^{-m(A) t}}{t^{k(d-1) / 2}}
$$

with $m(A)>0$ and $k$ a positive integer, then $A$ couples $\Omega$ to a state describing at most $k$ particles, whose total mass is $m(A)$.

In the next section, we establish behaviour (4.6) for the soliton two-point functions

$$
\left\langle S_{1}(0) \Omega, T(t) U(\mathbf{x}) S_{1}(0) \Omega\right\rangle
$$

of all the models introduced in Sect. 3.1, in the phase where $\left\langle\Omega, S_{1}(0) \Omega\right\rangle=0$. We also suggest that, for $A=S_{q}(x)$ with $q>1$, one may find a behaviour like (4.8), with $k=q$.

We close this section with a sketch of the proof that the mass gap

$$
m_{q}=\inf \left\{\operatorname{spec}\left(M \uparrow \mathscr{H}_{q}\right)\right\}
$$


of the soliton sector $\mathscr{H}_{q}, q \neq 0$, coincides with the decay rate of the two-point function of $S_{q}(x)$, i.e.

$$
m_{q}=\lim _{t>\infty}-\frac{1}{t} \ln \left\langle S_{q}(0) \Omega, S_{q}(t, \mathbf{0}) \Omega\right\rangle .
$$

The proof works for theories where Euclidean Green functions are given by expectations in a positive measure, i.e. for all our models without fermions. We first observe that $\mathscr{A} \Omega \cap \mathscr{H}_{q}$ is dense in $\mathscr{H}_{q}$, by construction. Moreover, every vector $A \Omega \in \mathscr{H}_{q}$ can be written as $S_{q}(0) A_{0} \Omega$ with $A_{0} \Omega \in \mathscr{H}_{0}$. Therefore, for some $A_{0} \Omega \in \mathscr{H}_{0}$,

$$
m_{q}=\lim _{t>\infty}-\frac{1}{t} \ln \left\langle S_{q}(0) A_{0} \Omega, T(t) S_{q}(0) A_{0} \Omega\right\rangle .
$$

Let $F_{A_{0}}$ denote the Euclidean field corresponding to $A_{0}$. Since $\mathscr{A}_{0} \Omega$ is dense in $\mathscr{H}_{0}$, we can choose $A_{0} \in \mathscr{A}_{0}$; with $\left\|F_{A_{0}}\right\|_{\infty}<\infty$. Hence

$$
\begin{aligned}
& \left\langle S_{q}(0) A_{0} \Omega, T(t) S_{q}(0) A_{0} \Omega\right\rangle=\left\langle\theta F_{A_{0}} \cdot\left(F_{A_{0}}\right)_{t} D(0,-q ; t, q)\right\rangle \\
& \quad \leqq\left\|F_{A_{0}}\right\|_{\infty}^{2}\langle D(0,-q ; t, q)\rangle=\left\|F_{A_{0}}\right\|_{\infty}^{2}\left\langle S_{q}(0) \Omega, T(t) S_{q}(0) \Omega\right\rangle .
\end{aligned}
$$

Therefore

$$
\begin{aligned}
& -\frac{1}{t} \ln \left\langle S_{q}(0) \Omega, T(t) S_{q}(0)\right\rangle \geqq m_{q}=\inf \left\{\operatorname{spec}\left(M \nmid \mathscr{H}_{q}\right)\right. \\
& \quad \geqq-\frac{1}{t} \ln \left\langle S_{q}(0) \Omega, T(t) S_{q}(0) \Omega\right\rangle,
\end{aligned}
$$

i.e. (4.9) is satisfied.

4.2. Excitation Analysis. To analyze the particle structure of the soliton sectors, we return to the representation of $\langle D(\omega)\rangle$ in terms of random lines given by Eq. (3.4). We discuss only the main ideas; precise definitions are deferred to Appendix 2.

We consider the two-point function of the soliton field operator $S_{q}(x)$. If $x$ is a point in the Euclidean space-time lattice with $x^{0}>0$, (3.4) reads

$$
\left\langle S_{q}(0) \Omega, S_{q}(x) \Omega\right\rangle=\sum_{v_{0 x}} z\left(v_{0 x}\right),
$$

where we have set $\left(v^{\omega}\right)^{*} \equiv v_{0 x}$.

For $q=1$, the support of $v_{0 x}$ is a single random line joining 0 to $x$, and $z\left(v_{0 x}\right)$ behaves, roughly speaking, like the statistical weight of a simple random walk from 0 to $x$. If $z\left(v_{0 x}\right)$ were exactly the weight of a simple random walk, then

$$
\sum_{v_{0 x}} z\left(v_{0 x}\right)=\operatorname{const}\left(-\Delta+m^{2}\right)^{-1}(0, x) \sim \frac{e^{-m|x|}}{|x|^{(d-1) / 2}},
$$

for some $m>0$. Hence we expect a behaviour

$$
\left\langle S_{1}(0) \Omega, S_{1}(x) \Omega\right\rangle \underset{|x| \rightarrow \infty}{\sim} \frac{e^{-m|x|}}{|x|^{(d-1) / 2}}, \quad m>0 .
$$


As remarked in the previous section, a decay like (4.12), i.e. an Ornstein-Zernike decay, implies that $S_{1}(x)$ couples the vacuum to a stable one-particle state of mass $m$.

For $q>1$, the support of $v_{0 x}$ consists, in general, of a set of $q$ distinct random lines, $\gamma_{i}$, carrying charge 1 . If the dominant contributions to (4.11) come from configurations where the lines $\gamma_{1}, \ldots, \gamma_{q}$ are essentially bound in one single line of charge $q$, then we would obtain

$$
\left\langle S_{q}(0) \Omega, S_{q}(x) \Omega\right\rangle \underset{|x| \rightarrow \infty}{\sim} \frac{e^{-m_{q}|x|}}{|x|^{(d-1) / 2}}, \quad 0<m_{q}<q m .
$$

Instead, if the dominant contributions come from configurations of $q$ independently fluctuating lines, each of charge 1 , then one expects that

$$
\left\langle S_{q}(0) \Omega, S_{q}(x) \Omega\right\rangle \underset{|x| \rightarrow \infty}{\sim} \frac{e^{-m_{q}|x|}}{|x|^{q(d-1) / 2}}, \quad m_{q}=q m>0 .
$$

In the first instance, $S_{q}(x)$ still couples $\Omega$ to a one-particle state, a bound state of $q$ solitons of charge 1 , in the second one, it couples $\Omega$ to a $q$-particle state. Both situations arise in concrete models. In the non-compact abelian Higgs model, for example, we expect that only (4.14) arises, whenever $|q|>1$, i.e. vortices do not bind.

For the $S U(N)$ Higgs model, with $N$ large and $q$ close to 1 or $N$, behaviour (4.14) is found.

We now review the basic method to establish behavior (4.6) in our models, for $A=S_{1}(x)$. In the region of coupling-constant space of a model where a C.L.H.T. expansion converges,

$$
\left|z\left(v_{0 x}\right)\right| \leqq e^{-C\left|v_{0 x}\right|}
$$

where $\left|v_{0 x}\right|$ is the length of the support of $v_{0 x}$, and $C$ is a large constant. The leading contribution to

$$
\sum_{\mathbf{x}} \sum_{\substack{v_{0 x} \\ x=(t, \mathbf{x})}} z\left(v_{0 x}\right)
$$

is then given by a single term, $z\left(\tilde{v}_{0 x}\right)$, where $\operatorname{supp} \tilde{v}_{0 x}$ is the straight line from 0 to $x=(t, \mathbf{0})$.

A link in the support of $v_{0 x}$ is called regular if it is in the $t$-direction and if there are no other links in $\operatorname{supp} v_{0 x}$ with the same projection onto the $t$-axis. If we omit from supp $v_{0 x}$ all the regular links, we are left with a set of connected lines, whose projection onto the $t$-axis are mutually disjoint. They are called excitations and denoted by $\varepsilon$. A path $v_{0 x}$ can be reconstructed from its excitations $\left\{\varepsilon_{1}, \ldots, \varepsilon_{n}\right\}$, and we may rewrite $z\left(v_{0 x}\right)$ as $Z\left(\varepsilon_{1}, \ldots, \varepsilon_{n}\right\}$.

We define an interaction, $U$, between the excitations by setting

so that

$$
U\left(\varepsilon_{1}, \ldots, \varepsilon_{n}\right)=\ln \left(\frac{Z(\emptyset)}{Z\left(\varepsilon_{1}, \ldots, \varepsilon_{n}\right)}\right)
$$

$$
\sum_{\mathbf{x}}\left\langle S_{1}(0) \Omega, S_{1}(t, \mathbf{x}) \Omega\right\rangle=\sum_{\mathbf{x}} \sum_{\substack{v_{0 x} \\ x=(t, \mathbf{x})}} z\left(v_{0 x}\right)=\sum_{\left\{\varepsilon_{1}, \ldots, \varepsilon_{n}\right\}} Z(\emptyset) e^{-U\left(\varepsilon_{1}, \ldots, \varepsilon_{n}\right)} .
$$


Equation (4.17) expresses the two-point function of the soliton field as a statistical sum of a one-dimensional gas of extended particles, the excitations, moving in the finite interval $[0, t]$. In the region where a C.L.H.T. expansion converges, this gas is dilute, and the strength of interactions between excitations is weak and decays exponentially in the distance. Hence the sum on the right-hand side of (4.17) can be exponentiated with the help of a convergent Mayer series, i.e.

$$
Z(\emptyset) \sum_{\left\{\varepsilon_{1}, \ldots, \varepsilon_{n}\right\}} e^{-U\left(\varepsilon_{1}, \ldots, \varepsilon_{n}\right\}} e^{t P[0, t]},
$$

where $P_{[0, t]}$ is the pressure of the gas of excitations constrained to the interval $[0, t]$.

The pressure $P_{[0, t]}$ can be expressed as the sum of three terms:

$$
t P_{[0, t]}=t P+P_{b d}+P_{\text {int }},
$$

where $P$ is the pressure in the thermodynamic limit, $P_{b d}$ is a correction due to the interaction of excitations with the boundaries of $[0, t]$, and $P_{\text {int }}$ is the interaction between the boundaries of $[0, t]$ mediated by the excitations. Now, $P_{\mathrm{nt}} \sim e^{-\mu t}$, as $t \nearrow \infty$, where $\mu$ is the correlation length of the one-dimensional gas of excitations. Hence

$$
\sum_{\mathbf{x}}\left\langle S_{1}(0) \Omega, S_{1}(t, \mathbf{x}) \Omega\right\rangle=e^{t P+P_{b d}+P_{\text {int }}} \underset{t>\infty}{\sim} \operatorname{const} e^{t P}\left(1+e^{-\mu t}\right) .
$$

By comparison with (4.6) we obtain

$$
m \equiv m\left(S_{1}\right)=-P, \quad \mu\left(S_{1}\right)=\mu .
$$

Using the excitation analysis and the estimates in Table 2 of Appendix 1, one can establish behaviour (4.20) for all our models and give estimates on $m\left(S_{1}\right)$ and $\mu\left(S_{1}\right)$. This analysis is made more precise in Appendix 2, where a derivation of estimates (4.21) for the kink in $\phi^{4}$, in two dimensions, is also sketched. For a more detailed discussion of the excitation analysis and estimates of $m\left(S_{1}\right)$ and $\mu\left(S_{1}\right)$ in our models, see [18]. (For background see [4].) We summarize the main results for the examples $\mathrm{A} 1-\mathrm{A} 5$ in the following Table 1 :

Table 1

A1 A2 A3

$\begin{array}{rlrl} & \beta, \lambda \text { large; } \beta / \lambda \text { small } & & \beta_{H}, \lambda \text { large } ; \beta_{F}, \beta / \lambda \text { small } \\ & \beta \gg|\ln \beta / \lambda| & \beta \gg|\ln \beta / \lambda|, \ln \lambda \\ m\left(S_{1}\right): & 2 \beta\left(1+0\left(\lambda^{-1 / 2}\right)\right) & 2 \beta\left(1+0\left(\lambda^{-1 / 2}\right)\right) \\ \mu\left(S_{1}\right): & 0(|\ln \beta| \lambda \mid) & & \min [0(|\ln \beta| \lambda \mid), 0(\ln \lambda)]\end{array}$

A4

$\beta_{G}, \beta_{H}$ large; $\beta_{G} / \beta_{H}$ small

$\beta_{G} \gg\left|\ln \beta_{G}\right| \beta_{H} \mid$

$m\left(S_{1}\right): \beta_{G}\left[\left(1-\cos \frac{2 \pi}{N}\right)+0\left(\beta_{H}^{-1 / 2}\right)\right]$

$\mu\left(S_{1}\right): \quad 0\left(\left|\ln \beta_{G} \beta_{H}^{-1}\right|\right)$
A3

$\beta_{G}, \beta_{H}, \lambda$ large; $\beta_{G} / \beta_{H} / \lambda$ small $\beta_{G} \gg\left|\ln \beta_{G} / \beta_{H}\right|,\left|\ln \beta_{H}\right| \lambda \mid$

$2 \pi^{2} \beta_{G}\left(1+0\left(\beta_{H}^{-1 / 2}\right)\right)$

$\min \left[0\left(\left|\ln \frac{\beta_{G}}{\beta_{H}}\right|\right), 0\left(\left|\ln \frac{\beta}{\lambda}\right|\right)\right]$

A5

$\beta_{G}, \beta_{H}$ large; $\beta_{G} / \beta_{H}$ small

$\beta_{G} \gg\left|\ln \beta_{G} / \beta_{H}\right|$

$2 \pi^{2} \beta_{G}\left(1+0\left(\beta_{H}^{-1 / 2}\right)\right)$

$0\left(\left|\ln \beta_{G} \beta_{H}^{-1}\right|\right)$ 


\section{Stringlike Solitons}

In this section, we discuss some examples of lattice field theories which exhibit stringlike soliton sectors and analyze some aspects of their correlation functions. Some characteristic features of the particle structure analysis on these sectors are outlined, too.

A two-dimensional example with stringlike solitons is obtained by perturbing a scalar theory with an even polynomial self-interaction, $\lambda \sum_{x} V\left(\phi_{x}\right)$, by a term $\beta_{B} \sum_{x} Q\left(\phi_{x}\right)$, where $Q$ is an odd polynomial with $\operatorname{deg} Q<\operatorname{deg} V$. Stringlike soliton sectors occur if this theory has two disjoint vacuum states not related by the symmetry $\phi \rightarrow-\phi[22]$; (example B1).

Three-dimensional models are obtained by adding to a gauge theory satisfying condition A, Sect. 2.3, a matter field with an action which is not invariant under the $\mathscr{Z}$-valued hypergauge transformations (2.22). For example, we can add Higgs or fermion fields with fractional charges to the non-compact abelian Higgs model (example B2), or fermions to the $S U(N)$ Higgs model (example B3).

A simpler model involving only discrete fields is the $\mathbb{Z}_{N}$-gauge theory with $\mathbb{Z}_{N}$-valued matter fields. Stringlike charged sectors in this model have been constructed by Fredenhagen and Marcu (F.M.) in [13].

In all these models we denote by $\beta_{B}$ the coupling parameter of the term of the action which is not hypergauge invariant.

As remarked in Sect. 2.3, the presence of a term in $S_{1}$ which is not hypergauge invariant makes the expectation value $\langle D(\omega)\rangle$ depend on $\omega$, and not just on $d \omega$.

As a consequence, a proof of O.S. positivity of disorder correlation functions similar to the one given for local solitons is not adequate. The support of $\omega^{*}$ has to be arranged in such a way that it does not intersect the time zero plane. Since we want $\operatorname{supp}(d \omega)^{*}$ to be given by a pre-assigned set of points $\left\{x_{i}\right\}$, this implies that $\operatorname{supp} \omega^{*}$ is in general non-compact. As we have seen in Sect. 2.3, correlation functions are defined by taking a thermodynamic limit of suitably normalized finite-lattice correlations. For the choice of $\omega$ made in Sect. 2.3, we choose a normalization factor $N_{A}$ constructed as follows. Let $\gamma_{x}$ denote a straight line of the dual lattice in the time direction connecting the bottom to the top of the lattice and intersecting $x$. Denote by $D\left(\gamma_{x_{1}}, q_{1}, \ldots, \gamma_{x_{r}}, q_{r}\right)$ the disorder field which corresponds to an $\omega$ satisfying

$$
\left(\omega^{*}\right)_{\langle x y\rangle}=q_{i}, \quad \text { for }\langle x y\rangle \in \gamma_{x_{i}}, \quad=0, \quad \text { otherwise. }
$$

We assume that $\Lambda=[-T, T] \times[-L, L]^{x(d-1)}$, and define

$$
\begin{aligned}
& N_{\Lambda}\left(\gamma_{x_{1}}^{-}, q_{1}, \ldots, \gamma_{x_{r}}^{-}, q_{r}, \gamma_{y_{1}}^{+}, q_{r+1}, \ldots, \gamma_{y_{s}}^{+}, q_{r+s}\right) \\
& \quad=\left\langle D\left(\gamma_{x_{1}}, q_{1}, \ldots, \gamma_{x_{r}}, q_{r}\right)\right\rangle_{\Lambda}^{1 / 2}\left\langle D\left(\gamma_{y_{1}}, q_{r+1}, \ldots, \gamma_{y_{s}}, q_{r+s}\right)\right\rangle_{\Lambda}^{1 / 2} .
\end{aligned}
$$

Let $\omega$ be as defined in Sect. 2.3 and $N_{A}$ as in (5.2). Let $O(\Gamma)$ denote an observable with local, connected support $\Gamma$. Then, by means of cluster expansions, one can prove the existence of the correlation functions

$$
\begin{aligned}
& S_{n=r+s, m}\left(\gamma_{x_{1}}^{-}, q_{1}, \ldots, \gamma_{x_{r}}^{-}, q_{r}, \gamma_{y_{1}}^{+}, q_{r+1}, \ldots, \gamma_{y_{s}}^{+}, q_{r+s} ; \Gamma_{1}, \ldots, \Gamma_{m}\right) \\
& \quad \equiv \lim _{\Lambda>\mathbb{Z}_{1 / 2}^{d}} N_{\Lambda}^{-1}\left(\gamma_{x_{1}}^{-}, q_{1}, \ldots, \gamma_{y_{s}}^{+}, q_{r+s}\right)\left\langle D\left(\gamma_{x_{1}}^{-}, q_{1}, \ldots, \gamma_{y_{s}}^{+}, q_{r+s}\right) O\left(\Gamma_{1}\right) \ldots O\left(\Gamma_{m}\right)\right\rangle_{\Lambda},
\end{aligned}
$$

for all models above. 
In the definition of the state $\langle(\cdot)\rangle_{\Lambda}$ in (5.2), (5.3) appropriate boundary conditions must be imposed: For example, in (5.3) $r$ strings $\gamma_{x_{1}}^{-}, \ldots, \gamma_{x_{r}}^{-}$end on the face $\{t=-T\}$ of $\partial \Lambda$, and $s$ strings $\gamma_{y_{1}}^{+}, \ldots, \gamma_{y_{s}}^{+}$end on the face $\{t=T\}$ of $\partial \Lambda$. Hence on $\{t=-T\}$ a total defect charge of $q=q_{1}+\ldots+q_{r} \in \mathscr{Z}$ is accumulated at the endpoints of the strings $\gamma_{x_{1}}^{-}, \ldots, \gamma_{x_{r}}^{-}$, while on $\{t=T\}$ a total defect charge of $-q=q_{r+1}+\ldots+q_{r+s}$ is accumulated. Therefore, one must impose a boundary field (of highest rank) on $\partial \Lambda$ corresponding to a defect current flowing from the face $\{t=-T\}$ to the face $\{t=T\}$, whose curl equals $q$ on $\{t=-T\}$, equals $-q$ on $\{t=T\}$ and vanishes elsewhere on $\partial \Lambda$.

In the example of the three-dimensional $\mathbb{Z}_{N}$-Higgs model, a path $\gamma$ is chosen on $\partial \Lambda$ which connects $\{t=-T\}$ to $\{t=T\}$, and one imposes the following boundary conditions on the gauge field, $g$, which is the field of highest rank in the model:

for all $b \in \partial \Lambda$.

$$
g_{b}= \begin{cases}q \in \mathbb{Z}_{N}, & \text { if } b^{*} \in \gamma \\ 1, & \text { if } b^{*} \notin \gamma,\end{cases}
$$

The O-S positivity of the correlation functions (5.3) is essentially obvious.

A simple way to prove the existence of the limits (5.3) is to apply a cluster expansion to both, numerator and denominator, and then exponentiate it by means of an excitation expansion. This method is discussed in detail in [18]. We now give a brief sketch of how it works in the $\mathbb{Z}_{N}$ Higgs model for the two-point function

$$
S_{2,0}\left(\gamma_{x}^{-},-1, \gamma_{y}^{+}, 1\right)=\lim _{\Lambda \succ \mathbb{Z}^{3}} \frac{\left\langle D\left(\gamma_{x}^{-},-1, \gamma_{y}^{+}, 1\right)\right\rangle_{\Lambda}}{N_{\Lambda}\left(\gamma_{x}^{-},-1, \gamma_{y}^{+}, 1\right)} .
$$

We work in the low-temperature phase and use the Marra-Miracle-Solé cluster expansion [23].

A configuration of the cluster expansion for $\left\langle D\left(\gamma_{x}^{-},-1, \gamma_{y}^{+}, 1\right)\right\rangle_{A}$ consists of a vortex line $\left(v^{\omega}\right)^{*}$ in the dual lattice joining $x$ to $y$ and a set of vortex loops and Wilson loops linked to $\left(\gamma_{x}^{-} \cup \gamma_{y}^{+}\right) \Delta \operatorname{supp}\left(v^{\omega}\right)^{*} ;(\Delta \equiv$ symmetric difference). A configuration of the cluster expansion for $\left\langle D\left(\gamma_{x}, \pm 1\right)\right\rangle_{1}$ consists of a set of vortex loops and Wilson loops linked to $\gamma_{x}$.

We now apply to both numerator and denominator, an excitation analysis; (see Sect. 4.2 and Appendix 2). The excitations in the numerator can be divided into two classes, the string excitations, $\varepsilon^{s}$, whose projections onto the time axis are contained in $\left(-\infty, x^{0}\right] \cup\left[y^{0},+\infty\right)$, and the particle excitations, $\varepsilon$, arising from the fluctuations of $v^{\omega}$, having non-empty projections in $\left[x^{0}, y^{0}\right]$ (see Fig. 4).

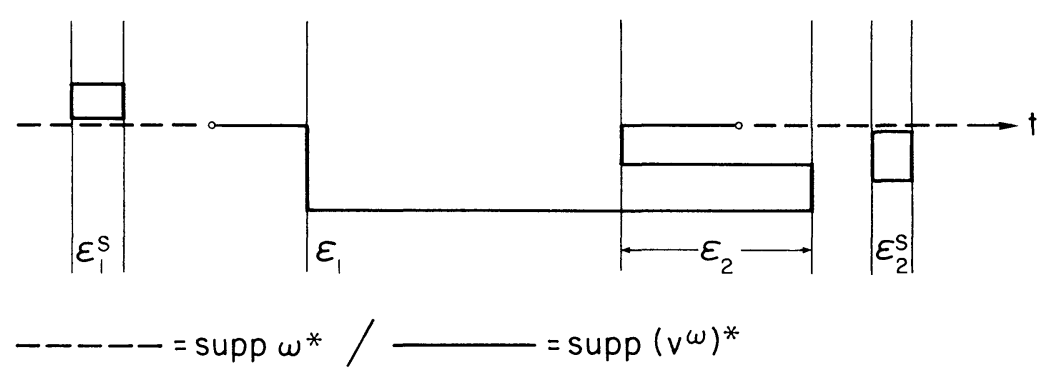

Fig. 4 
In the denominator only string excitations appear. When we exponentiate the expansion, the contributions coming from string excitations in the numerator are essentially cancelled by the contributions due to the string excitations of the denominator. Hence all clusters of excitations left have non-empty projections onto the time axis contained in $\left[x^{0}, y^{0}\right]$, and one can now easily prove the existence of the thermodynamic limit.

Clustering of correlation functions and vanishing of correlation functions with non-zero total charge easily follow by noticing that the contribution of every vortex line is exponentially decreasing in the total length of the vortex line.

Hence, by Theorem 2.3 , one can construct $\mathbb{Z}_{N}$ soliton (vortex) sectors in the low-temperature phase of the 3-D $\mathbb{Z}_{N}$ Higgs model. The particle structure of these sectors can be analyzed with the help of an excitation expansion, as in Sect. 4.2 and Appendix 2. The result of this analysis is a decay law

$$
\sum_{\mathbf{x}} S_{2}\left(\gamma_{0}^{-},-1, \gamma_{x}^{+}, 1\right) \underset{t \rightarrow+\infty}{\sim} \text { const } e^{-\left[\beta_{G}(1-\cos (2 \pi / N))+O\left(\ln \beta_{B}\right)\right] t} \cdot\left(1+e^{-O\left(\ln \beta_{B} \mid\right) t}\right),
$$

for $\beta_{G}$ large, $\beta_{B}$ small, $\beta_{G} \gg\left|\ln \beta_{B}\right| \gg \ln \beta_{G}$. This proves that the soliton field operator $S_{1}\left(\gamma_{x}^{+}\right)$couples the vacuum to a stable one-particle state.

Using the excitation expansion, one can also show that, since the model has short range interactions, joint expectations of local observables and disorder fields are independent of the choice of the asymptotic direction of the strings $\gamma_{x_{1}}$. The independence of the soliton states of the direction of the strings of the solitons is expected on the basis of the axiomatic analysis of non-local charged states in massive theories performed in [24].

Remark 5.1. The $\mathbb{Z}_{N}$ Higgs model in $d$ dimensions is dual to a rank- $k \mathbb{Z}_{N}$ Higgs model with $k=d+2$ and coupling constants $\beta_{G}^{*}=0\left(\frac{1}{\beta_{B}}\right), \beta_{B}^{*}=0\left(\frac{1}{\beta_{G}}\right)$. The duality transformation maps the F-M string observable onto the disorder field discussed above.

Fredenhagen and Marcu [13] constructed a charged state in the $\mathbb{Z}_{N}$ model, for small $\beta_{B}$ and large $\beta_{G}$, making use of a sequence of string fields with one end of the string tending to $\infty$. The soliton state we construct in $d=3$ is the dual of this charged state. More generally, vortex sectors in $d=k+2$ dimensions in rank- $k$ $\left(\mathbb{Z}_{N}\right.$-Higgs) models - which are constructed just like the vortex sectors in the three-dimensional $\mathbb{Z}_{N}$-Higgs model - are the duals of their charged sectors.

In $d=3$ dimensions, the $\mathbb{Z}_{N}$-Higgs model is self-dual. We may therefore consider mixed correlation functions of disorder fields, local order fields and of the charged fields of Fredenhagen and Marcu. Applying Theorem 2.3 to such mixed correlation functions, we can prove that in the phase corresponding to large $\beta_{G}$ and small $\beta_{B}$ the Hilbert space $\mathscr{H}$ of physical states decomposes into a direct sum, $\underset{q_{1}, q_{2}}{\bigoplus} \mathscr{H}_{q_{1}, q_{2}}$, of sectors, $\mathscr{H}_{q_{1}, q_{2}}$, labelled by an "electric charge," $q_{1}$ (the charge of Fredenhagen and Marcu) and a defect charge $q_{2}$ (the vorticity). These charges take values in $\mathbb{Z}_{N}$. This is expected to be a complete description of the super-selection structure of the threedimensional $\mathbb{Z}_{N}$-Higgs model.

An analysis analogous to the one just sketched for the $\mathbb{Z}_{N}$-Higgs model can be performed for the examples B1-3, using a kind of C.L.H.T. expansion; (see [18]). 
In particular, one can prove the existence of $\mathbb{Z}_{2}$ kink sectors for the example B1, for $\beta_{B}$ small, $\beta, \lambda$ large, and of $\mathbb{Z}_{N}$ vortex sectors for the examples $\mathrm{B} 2$ and $\mathrm{B} 3$, for $\beta_{B}$ small, $\beta_{G}, \beta_{H}$ large. Moreover, in this parameter region, the Ornstein-Zernike decay of the two-point correlation function for the soliton field $S_{1}\left(\gamma_{x}\right)$ can be obtained. These results show that the existence of stringlike soliton sectors is not limited to models in which the basic fields are discrete, nor to the existence of a duality transformation. We therefore expect that there are continuum theories with stringlike soliton sectors, such as the three-dimensional $S U(N)$ Higgs models with fractionally charged fermions.

\section{The Monopole Sectors in the $U(1)_{4}$ Gauge Theory}

The partition function of the $U(1)_{4}$ gauge theory in the Villain form is given by

$$
Z=\int \prod_{\langle x y\rangle} d \theta_{\langle x y\rangle} \sum_{n} e^{-(\beta / 2)\|d \theta+2 \pi n\|^{2}},
$$

where $\theta$ is a $U(1)$-valued gauge field, $n$ is a second rank, $\mathbb{Z}$-valued field, and $\|\cdot\|$ is the norm determined by the scalar product (2.4). We first recall a representation of the expectation value of the disorder field in terms of magnetic currents. In Sect. 2.3, the disorder field $D(\omega, B)$ has been defined by

$$
\langle D(\omega, B)\rangle=\frac{1}{Z} \int \prod_{\langle x y\rangle} d \theta_{\langle x y\rangle} \sum_{n} e^{-(\beta / 2)\left\|d \theta+2 \pi n+2 \pi \delta \Delta^{-1}(B-\omega)\right\|^{2}},
$$

where $\omega$ is a third rank, $\mathbb{Z}$-valued field with $\operatorname{supp}(d \omega)^{*}=\left\{x_{i}\right\}$ and $(d \omega)^{*}\left(x_{i}\right)$ $=q_{i} \in \mathbb{Z} \backslash\{0\} ; B$ is a classical magnetic field given by

$$
B=\sum_{i} B\left(x_{i}, q_{i}\right),
$$

where $(d B)^{*}\left(x_{1}, q_{i}\right)=q_{i} \delta_{x_{i}}$, so that $d(\omega-B)=0$, and $B\left(x_{i}, q_{i}\right)$ is a "Coulomb-like" classical field, with support in a constant-time plane, see Sect. 2.3. We use the Hodge decomposition to rewrite

$$
n=d \Delta^{-1} \delta n+\delta \Delta^{-1} m,
$$

where

$$
m=d n \text {. }
$$

Let $n[m]$ be an integer-valued solution of the cohomological equation (6.4). Then every other solution is of the form $n[m]+d l$, with $l$ a $\mathbb{Z}$-valued field of rank 1 .

We can now rewrite the numerator of (6.2) as

$$
\sum_{m: d m=0} \sum_{l} \int \prod_{\langle x y\rangle} d \theta_{\langle x y\rangle} e^{-(\beta / 2)\left\|d\left[\theta+2 \pi l+2 \pi \delta \Delta^{-1} n[m]\right]+2 \pi \delta \Delta^{-1}[m+B-\omega]\right\|^{2}} .
$$

Let us define a real-valued gauge field $A$ by setting

$$
A=\theta+2 \pi l+2 \pi \delta \Delta^{-1} n[m] .
$$


Note that the cross term

$$
\left(d A, \delta \Delta^{-1}(m+B-\omega)\right)
$$

in the exponent of the integrand of (6.5) vanishes. Moreover

$$
\left(\delta \Delta^{-1}(m+B-\omega), \delta \Delta^{-1}(m+B-\omega)\right)=\left(m+B-\omega, \Delta^{-1}(m+B-\omega)\right),
$$

since $d(m+B-\omega)=0$.

Hence $\langle D(\omega, B)\rangle$ is given by

$$
\begin{aligned}
\langle D(\omega, B)\rangle & =\frac{\sum_{m: d m=0} \int \prod_{\langle x y\rangle} d A_{\langle x y\rangle} e^{-(\beta / 2)\|d A\|^{2}} e^{-(\beta / 2)\left(m+B-\omega, \Delta^{-1}(m+B-\omega)\right)}}{\sum_{m: d m=0} \int \prod_{\langle x y\rangle} d A_{\langle x y\rangle} e^{-(\beta / 2)\|d A\|^{2}} e^{-(\beta / 2)\left(m, \Delta^{-1} m\right)}} \\
& =\frac{\sum_{m: d m=-d B} e^{-(\beta / 2)\left(m+B, \Delta^{-1}(m+B)\right)}}{\sum_{m+d m=0} e^{-(\beta / 2)\left(m, \Delta^{-1} m\right)}},
\end{aligned}
$$

where $\int \prod_{\langle x y\rangle} d A_{\langle x y\rangle}$ denotes integration over the gauge equivalence classes of the gauge field $A$. The dual of the field $m, m^{*}$, is supported on a set of lines in the dual lattice which may be interpreted as magnetic current lines. They are the defects of the $U(1)_{4}$ gauge theory. Equation (6.6) justifies our definition (2.29), (2.30) of $D(\omega, B)$.

Applying the Jensen inequality to (6.6) one obtains the lower bound

$$
\langle D(\omega, B)\rangle \geqq e^{-(\beta / 2)\left(B-\omega, \Delta^{-1}(B-\omega)\right)},
$$

which shows that $\langle D(\omega, B)\rangle$ does not vanish, as long as $\left(B-\omega, \Delta^{-1}(B-\omega)\right)<\infty$.

We now turn to the proof of O-S positivity of the correlation functions (2.33). The easiest way to prove O-S positivity is to apply a duality transformation which we now discuss for the special case of $\langle D(\omega, B)\rangle$. The analysis of more general expectations is straightforward.

We start by performing a Fourier transformation in the variable $(d \theta)_{p}$, given by

$$
\sum_{n} e^{-(\beta / 2)\left\|d \theta+2 \pi n+2 \pi \delta \Delta^{-1}(B-\omega)\right\|^{2}}=\sum_{n} e^{l\left(n, d \theta+2 \pi \delta \Delta^{-1}(B-\omega)\right)} e^{-(\beta / 2)\|n\|^{2}} .
$$

Integrating over $\theta$, we arrive at the following constraint for the field $n$ :

$$
\delta n=0 .
$$

Next, we pass to the dual lattice and solve the constraint equation (6.8), using the Poincare lemma, $n^{*}=d A$, where $n^{*}$ is the field dual to $n$, and $A$ is a $\mathbb{Z}$-valued gauge field. Let $[A]$ denote the gauge-equivalence class represented by $A$. Then we may rewrite $(6.2)$ as

$$
\langle D(\omega, B)\rangle=\frac{\sum_{[A]} e^{-1 /(2 \beta)\|d A\|^{2}} e^{l 2 \pi\left(d A, d \Delta^{-1}(B-\omega)^{*}\right)}}{\sum_{[A]} e^{-1 /(2 \beta)\|d A\|^{2}}} .
$$


Using the identity

$$
\delta d \Delta^{-1}(B-\omega)^{*}=B^{*}-\omega^{*},
$$

and noticing that, since $\omega^{*}$ and $A$ are integers, $e^{i 2 \pi\left(A, \omega^{*}\right)}=1$, we obtain

$$
\langle D(\omega, B)\rangle=\frac{1}{Z} \sum_{[A]} e^{-1 /(2 \beta)\|d A\|^{2}} e^{i 2 \pi\left(A, B^{*}\right)} \equiv\left\langle e^{i 2 \pi\left(A, B^{*}\right)}\right\rangle^{*} .
$$

Let $\left\{x_{1}, x_{2}\right\}=\operatorname{supp}(d \omega)^{*}$ and $B=B\left(x_{1}, q\right)+B\left(x_{2},-q\right)$. The classical fields $B^{*}\left(x_{1}, q\right)$ and $B^{*}\left(x_{2},-q\right)$ are supported in the planes $\left\{x^{0}=x_{1}^{0}\right\},\left\{x^{0}=x_{2}^{0}\right\}$ respectively. Choosing $x_{1}, x_{2}$ such that $x_{1}^{0}>0>x_{2}^{0}$, it is clear that

$$
e^{i 2 \pi\left(A, B^{*}\right)}=\theta\left[e^{i 2 \pi\left(A, B^{*}\left(r x_{2}, q\right)\right)}\right] e^{i 2 \pi\left(A, B^{*}\left(x_{1}, q\right)\right)}
$$

O-S positivity of $\langle D(\omega, B)\rangle$ is an immediate consequence of (6.10), (6.11).

Next we discuss the behaviour of $\langle D(\omega, B)\rangle$ in the two phases of the $U(1)_{4}$ gauge model, the confining and the Q.E.D. phase. The confining phase of the $U(1)_{4}$ model corresponds to the superconducting phase of the dual model derived in (6.10). This phase is massive, and one can show with the help of a simple lowtemperature expansion that

$$
\langle D(\omega, B)\rangle=\left\langle e^{2 \pi i\left(A, B^{*}\right)}\right\rangle^{*} \geqq e^{-\left(1 / 2 \beta^{\prime}\right)\left(B^{*},\left(\Delta+m_{A}^{2}\right)^{-1} B^{*}\right)} \geqq \text { const }
$$

uniformly in $\left|x_{1}-x_{2}\right|$. Here $\beta^{\prime}$ and $m_{A}^{2}$ are strictly positive constants which can be estimated as in [19].

We now turn to an analysis of $\langle D(\omega, B)\rangle$ in the Q.E.D. phase of the $U(1)_{4}$ model.

Clustering in the Q.E.D. phase and vanishing of correlation functions of nonzero total charge can be proved in the dual model by means of correlation inequalities as in [25]. However, for later discussions we need a more refined upper bound.

This has been obtained in $[1,17]$ using a suitable combination of the Peierls argument with renormalization group techniques.

Here we only summarize the results for $S_{2}(x, 1, y,-1)$. The discussion can easily be generalized to all the other correlation functions. Let us be somewhat more general than in Sect. 2.3 and take two different shapes $B_{0}$ and $B_{0}^{\prime}$ for the fields at $x$ and $y$, i.e.

$$
B=\left(B_{0}\right)_{x}-\left(B_{0}^{\prime}\right)_{y}
$$

To make the dependence on the shape of the magnetic field explicit, we rewrite the two-point function $S_{2}\left(x, 1, B_{0}, y,-1, B_{0}^{\prime}\right)$ in a more convenient form. By using the representation in terms of magnetic currents we can write

$$
S_{2}\left(x, 1, B_{0}, y,-1, B_{0}^{\prime}\right)=\frac{\sum_{\left\{m, m_{D}\right\}} e^{-(\beta / 2)\left(m+m_{D}+B, \Delta^{-1}\left(m+m_{D}+B\right)\right)}}{\sum_{\{m\}} e^{-(\beta / 2)\left(m, \Delta^{-1} m\right)}},
$$

where $m$ denotes a set of currents satisfying $d m=0$, and $m_{D}$ is a current satisfying $d m_{D}=-d B=-d \omega$, dual to a line joining $x$ to $y$. 
From $[1,17]$ it follows that, at large distances, the main effect of the closed currents, $m$, is just to renormalize the coupling constant $\beta$. Hence, as $|x-y| \nearrow \infty$,

$$
S_{2}\left(x, 1, B_{0}, y,-1, B_{0}^{\prime}\right) \sim \sum_{m_{D}} e^{-\left(\beta_{\mathrm{ren}} / 2\right)\left(m_{D}+B, \Delta^{-1}\left(m_{D}+B\right)\right)},
$$

where $\beta_{\text {ren }}=\beta-e^{-0(\beta)}$.

Equation (6.14) embodies several pieces of information: Clustering and vanishing of correlation functions of non-zero total charge easily follow from (6.14) and from its generalization to the other correlation functions. If we take, for all correlation functions, a fixed choice, $B_{0}$, of the magnetic field, $\mathbb{Z}$-monopole sectors can then be constructed in the Q.E.D. phase using Theorem 2.3. In particular, monopole field operators $S_{q}\left(x, B_{0}\right)$ localized in $\operatorname{supp}\left(B_{0}\right)_{x}$ are obtained. The sectors dual to the monopole sectors are the charged sectors of the $\mathbb{Z}$-gauge model described by the vacuum functional $\langle(\cdot)\rangle^{*}$, and can be viewed as limits of the charged sectors of the non-compact abelian Higgs model, (as $\lambda \nearrow \infty$; see [1]).

From the explicit construction one derives that the monopole sectors $\mathscr{H}_{q}, q \neq 0$, depend on the choice of the shape, $B_{0}$, of the magnetic field. Hence we denote them, more explicitly, by $\mathscr{H}_{q}\left(B_{0}\right)$. It is natural to ask if $\mathscr{H}_{q}\left(B_{0}\right)$ is orthogonal to $\mathscr{H}_{q}\left(B_{0}^{\prime}\right)$, for $B_{0} \neq B_{0}^{\prime}$. The scalar product between states $\left|x, 1, B_{0}\right\rangle \in \mathscr{H}_{1}\left(B_{0}\right)$ and $\left|y, 1, B_{0}^{\prime}\right\rangle \in \mathscr{H}_{1}\left(B_{0}^{\prime}\right)$ is given by

$$
S_{2}\left(r x,-1, B_{0}, y, 1, B_{0}^{\prime}\right) .
$$

From the upper bound (6.14) one obtains that

$$
\left\langle x, 1, B_{0} \mid y, 1, B_{0}^{\prime}\right\rangle=0,
$$

if

$$
\left(B(r x,-1)+B^{\prime}(y, 1), \Delta^{-1}\left(B(r x,-1)+B^{\prime}(y, 1)\right)\right)
$$

diverges.

This happens, for example, if $B_{0}$ and $B_{0}^{\prime}$ are localized in different spatial cones $\mathscr{C}, \mathscr{C}^{\prime}$ with apex in 0 .

The states obtained from fields $B$ localized in cones are the lattice approximations of the states discussed by Buchholz for Q.E.D. in [26]. In particular, if we choose $\mathscr{C}^{\prime}$ to be a cone obtained from $\mathscr{C}$ by a rotation, then the vanishing of (6.15) shows that, in the continuum limit, rotations cannot be unitarily implemented on Buchholz states.

We now turn to an analysis of the particle structure suggested by (6.14), for $B_{0}=B_{0}^{\prime}$. The contribution coming from the fluctuating current line $m_{D}$ is expected to produce an Ornstein-Zernike decay corresponding to a massive particle. However, there is also the contribution of the $B$-field, and this gives a power correction to the exponential decay:

$$
\exp -\beta_{\text {ren }}\left(B, \Delta^{-1} B\right) \sim|x-y|^{-\bar{c} \beta_{\text {ren }}}, \bar{c}>0 .
$$

Therefore one expects

$$
S_{2}(x,-1, y, 1) \underset{\left|x^{0}-y^{0}\right| \gamma_{\infty}}{\sim} \frac{e^{-c \beta_{\mathrm{ren}}\left|x^{0}-y^{0}\right|}}{\left|x^{0}-y^{0}\right|^{(d-1 / 2)+\bar{c} \beta_{\mathrm{ren}}}} .
$$


Equation (6.17) exhibits the infraparticle nature of the monopole. It shows that the mass operator $M$ does not have a discrete eigenvalue corresponding to the monopole state $S_{1}\left(x, B_{0}\right) \Omega$. This is due to the "cloud" of soft photons, surrounding the monopole, which produces the power correction (6.16) to the Ornstein-Zernike decay. See also [28].

We expect that all these features of the magnetic monopole in the $U(1)_{4}$ gauge theory are found in the analysis of all monopole sectors, e.g. in the 't HooftPolyakov monopole sectors in non-abelian gauge theories, but we have no rigorous results, so far.

\section{Appendix 1. The C.L.H.T. Expansion}

In this appendix we give an outline of the C.L.H.T. expansion for the disorder correlation functions of our models A1-5). Details appear in $[17,18]$.

For $d \geqq 3$, we eliminate, in a preliminary step, the "angular" degrees of freedom of the matter field by imposing the unitary gauge. The advantage is that, in this gauge, the Higgs mechanism which renders the gauge field massive appears in its clearest form. The field corresponding to the surviving radial degrees of freedom is taken to be one of the orbit fields of Sect. 3.3, to which the high temperature cluster expansion is applied.

The first step of the C.L.H.T. expansion is to rewrite the partition function, $Z$, of the model as the partition function of a polymer gas with polymers of two different types: $v$-polymers and $X$-polymers.

A $v$-polymer arises in the low temperature expansion for the $v$-field. It has support on a set of cells whose dual is connected and closed.

An $X$-polymer arises in the high-temperature expansion for the remaining (orbit) fields and has support on a connected set of cells (see Fig. A1).

The activity of a $v-(X-)$ polymer is denoted by $z(v)(z(X))$, and the partition function, $Z$, can be rewritten as

$$
Z \simeq \text { const } \sum_{\left\{X_{1} \ldots X_{n}\right\}} \sum_{\left\{v_{1} \ldots v_{m}\right\}} \prod_{i=1}^{n} z\left(X_{i}\right) \prod_{j=1}^{m} z\left(v_{j}\right),
$$

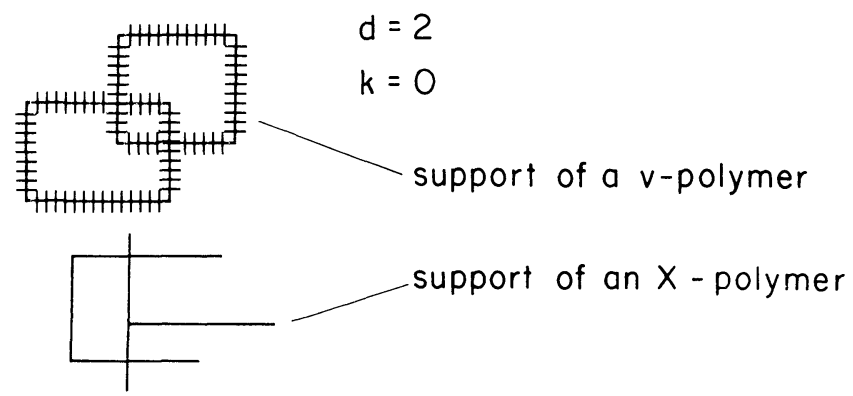

Fig. A1 
where $\left\{X_{1} \ldots X_{n}\right\}$ denotes a set of $x$-polymers with disjoint supports and $\left\{v_{1} \ldots v_{m}\right\}$ a set of $v$-polymers such that the dual of their supports are disjoint.

To be more concrete, consider the example A1),

$$
\begin{aligned}
Z \simeq \text { const } & \sum_{\left\{v_{1} \ldots v_{m}\right\}} \sum_{\left\{X_{1} \ldots X_{n}\right\}} \prod_{j=1}^{m} e^{-2 \beta\left|v_{j}\right|} \\
& \cdot \prod_{i=1}^{n}\left[\left\{\int \prod_{x \in X_{i}} d \varrho_{x} e^{-\lambda\left(\varrho_{x}^{2}-1\right)^{2}} \prod_{\langle x y\rangle X_{i}} \exp -\beta\left[\left(\varrho_{x}-e^{i \pi v\langle x y\rangle} \varrho_{y}\right)^{2}-\left(1-e^{i \pi v\langle x y\rangle}\right)^{2}\right]\right\}\right. \\
& \left.\cdot\left\{\int \prod_{x \in X_{i}} d \varrho_{x} e^{-\lambda\left(\varrho_{x}^{2}-1\right)^{2}}\right\}^{-1}\right],
\end{aligned}
$$

where $\left|v_{j}\right|=\operatorname{card}\left(\operatorname{supp} v_{j}\right)$.

The modified partition function in the numerator of (3.2), $Z(\omega)$, contains, besides ordinary $v$ - and $X$-polymers, a $v^{\omega}$-polymer such that every connected component of $\operatorname{supp}\left(v^{\omega}\right)^{*}$ has non-vanishing intersection with $\operatorname{supp}(d \omega)^{*}$, and $d v^{\omega}$ $=d \omega$. Hence,

$$
Z(\omega) \simeq \text { const } \sum_{\left\{X_{1} \ldots X_{n}\right\}} \sum_{\left\{v_{1} \ldots v_{m}, v^{\omega}\right\}} \prod_{j=1}^{n} z\left(X_{j}\right) \prod_{i=1}^{m} z\left(v_{i}\right) z\left(v^{\omega}\right),
$$

where, again, $\operatorname{supp}\left(v^{\omega}\right)^{*} \cap \operatorname{supp}\left(v_{i}\right)^{*}=\emptyset$.

Now we combine $v$-polymers and $X$-polymers in $Z$ into "connected" clusters, $C$; (see e.g. [27]). A configuration $\left\{v_{1}, \ldots, v_{m}, X_{1} \ldots X_{n}\right\}$ is said to form a cluster on

$$
C=\operatorname{supp}\left[\left(\bigcup_{j=1}^{m} v_{j}\right) \cup\left(\bigcup_{i=1}^{n} X_{i}\right)\right]
$$

iff supp $\left[\left(\bigcup_{j=1}^{m}\left(v_{j}\right)^{*}\right) \cup\left(\bigcup_{i=1}^{n} X_{i}\right)\right]$ is connected (see Fig. A.2).

The activity of the cluster $C$ is defined by

$$
Z(C)=\sum_{\substack{\left\{v_{1} \ldots v_{m}\right\}\left\{X_{1} \ldots X_{n}\right\} \\ \text { clusters on } C}} \prod_{j=1}^{m} z\left(v_{j}\right) \prod_{i=1}^{n} z\left(X_{i}\right) .
$$

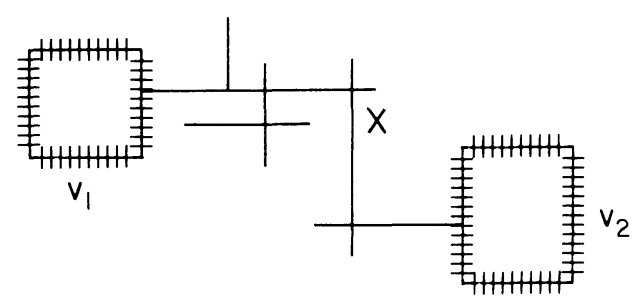

Fig. A2. $A$ configuration $\left(v_{1}, v_{2}, X\right)$ forming a cluster on $C=\operatorname{supp}\left(v_{1} \cup v_{2} \cup X\right) ; d=2, k=0$ 
In the expression for $Z(\omega)$ a cluster, $C^{\omega}$, containing $v^{\omega}$, arises, in addition to the usual clusters $C$. A configuration $\left\{v_{1}, \ldots, v_{m}, v^{\omega}\right\},\left\{X_{1}, \ldots, X_{n}\right\}$ is said to form a cluster on

$$
C^{\omega}=\operatorname{supp}\left[\left(\bigcup_{j=1}^{m} v_{j}\right) \cup\left(\bigcup_{i=1}^{n} X_{i}\right) \cup v^{\omega}\right]
$$

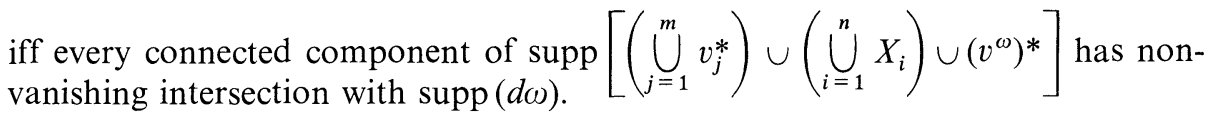

The activity of $C^{\omega}$ is given by

$$
Z\left(C^{\omega}\right)=\sum_{\substack{\left\{v_{1} \ldots v_{m}, v^{\omega}\right\} \\ \text { clusters on } C^{\omega}}} \prod_{j=1}^{m} z\left(v_{j}\right) \prod_{i=1}^{n} z\left(X_{i}\right) z\left(v^{\omega}\right) .
$$

Using standard methods [12]; one can write

$$
\langle D(\omega)\rangle=\frac{Z(\omega)}{Z}=\left.\frac{d}{d \alpha} \log (Z+\alpha Z(\omega))\right|_{\alpha=0}=\sum_{\left\{\mathbf{C}, C^{(\omega)}\right\}} a_{T}\left(\mathbf{C}, C^{\omega}\right) \prod_{C \in \mathbf{C}} Z(C) Z\left(C^{\omega}\right),
$$

where $\left\{\mathbf{C}, C^{\omega}\right\}$ denotes a collection of clusters in which a cluster $C$ can occur an arbitrary number of times and a cluster $C^{\omega}$ occurs once, and $a_{T}$ is the combinatiorial factor appearing in Eq. (3.5).

Equation (A.3) is the generalization of (3.4), (3.5) to models with continuous fields.

Theorem A.1. The expansion (A.4) for the expectation value of the disorder field $\langle D(\omega)\rangle$ converges if every cluster activity is dominated by an exponentially decreasing factor, i.e.

$$
|Z(C)| \leqq e^{-K|C|}
$$

with $K$ large enough and $|C|=\operatorname{card} C$.

Moreover, if we denote by $v_{\min }^{\omega}$ the configuration of $v^{\omega}$ with the highest activity and if

$$
\left|Z\left(C^{\omega}\right)\right| \leqq e^{-K\left|C^{\omega}\right|} e^{-K_{1}\left|v_{\min }^{\omega}\right|},
$$

\begin{tabular}{|c|c|c|c|}
\hline A 1) & & A2) & A3) \\
\hline K & $0(|\ln \beta / \lambda|)$ & $\min [0(|\ln \beta / \lambda|), 0(\ln \lambda)]$ & $\min \left[0\left(\mid \ln \frac{\beta_{G}}{\beta_{H}}\right)\right), 0(|\ln \beta / \lambda|)$ \\
\hline \multirow[t]{4}{*}{$K_{1}$} & $2 \beta$ & $2 \beta$ & $0\left(\beta_{G}\right)$ \\
\hline & A4) & & A5) \\
\hline & $K$ & $0\left(\left|\ln \beta_{G} / \beta_{H}\right|\right)$ & $0\left(\left|\ln \beta_{G} / \beta_{H}\right|\right)$ \\
\hline & $K_{1}$ & $0\left(\beta_{G}\right)$ & $0\left(\beta_{G}\right)$ \\
\hline
\end{tabular}

then clustering holds for all correlation functions, and the limits (2.14) vanish.

The constants $K$ and $K_{1}$ in our examples A1-5) are summarized in Table 2.

Table 2. (The conditions of Table 1 are assumed for the coupling constant) 
Equation (3.4) and bound (3.5) are obtained by partially resumming (A3), (A4) and using (A5), (A6).

\section{Appendix 2. Estimates for $m\left(S_{i}\right)$ and $\left(S_{j}\right)$}

In this appendix we present some details concerning the excitation analysis of Sect. 4.2, using the C.L.H.T. expansion of Appendix 1. From (A.4) it follows that

$$
\left\langle S_{1}(0) \Omega, S_{1}(x) \Omega\right\rangle=\sum_{\left\{\mathbf{C}, C_{0 x}\right\}} a_{T}\left(\mathbf{C}, C_{0 x}\right) Z\left(C_{0 x}\right) \prod_{C \in \mathbf{C}} Z(C),
$$

where we have set $C^{\omega}=C_{0 x}$ and $x=(t, \mathbf{x})$.

In this case $C_{0 x}$ is given by the dual of a line directed from 0 to $x$, corresponding to $\operatorname{supp} v^{\omega}$, enriched by cells coming from the support of $v$-and $X$-polymers in $C_{0 x}$.

By the explicit form of the combinational factor $a_{T}$, every configuration $\left\{\mathbf{C}, C_{0 x}\right\}$ contributing to (A.7) is given by a cluster $C_{0 x}$, enriched by a set of clusters $C$ in C "connected" to $C_{0 x}$. Hence it appears as a "decorated" path from 0 to $x$; (see Fig. A3)

We now define the excitations.

A cell in $\left(\mathbf{C}, C_{0 x}\right)$ is defined to be regular if

1) it is orthogonal to the time axis,

2) it occurs only in $C_{0 x}$,

3) there are no other cells having the same projection onto the time axis,

4) it is not connected to other cells.

The "connected" complements of the regular cells in $\mathbf{C}, C_{0 x}$ are the excitations, $\varepsilon$. Their projection on to the time axis are denoted by $\pi(\varepsilon)$. If $\varepsilon_{1} \ldots \varepsilon_{n}$ are the excitations corresponding to the configuration $\mathbf{C}, C_{0 x}$, then we set

$$
Z\left(\varepsilon_{1} \ldots \varepsilon_{n}\right)=a_{T}\left(\mathbf{C}, C_{0 x}\right) Z\left(C_{0 x}\right) \prod_{C \in \mathbf{C}} Z(C) .
$$

Since the configuration corresponding to $Z(\emptyset)$ is the dual of a straight line, all the cells contributing are disjoint. Let $z_{\omega}\left(c_{k+1}\right)$ denote the contribution to $Z(\emptyset)$ of one of these cells, then

$$
Z(\emptyset)=z_{\omega}\left(c_{k+1}\right)^{t}
$$

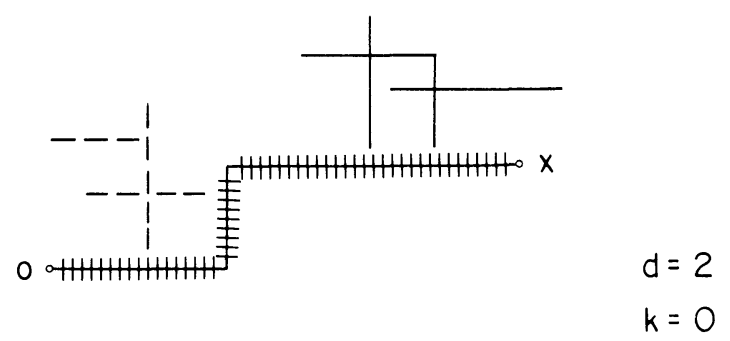

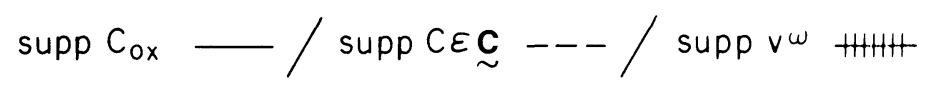

Fig. A3 
Inspection of the explicit form of $C_{0 x}, \mathbf{C}$ easily shows that the following factorization property holds for all our models: There exists a function $\zeta$ on the space of excitations such that

$$
\frac{Z\left(\varepsilon_{1} \ldots \varepsilon_{n}\right)}{Z(\emptyset)}=\left\{\begin{array}{lll}
\prod_{i=1}^{n} \zeta\left(\varepsilon_{i}\right) & \text { if } \pi\left(\varepsilon_{i}\right) \cap \pi\left(\varepsilon_{j}\right)=\emptyset, & \\
0, & \text { otherwise. } & \text { for all } i, j
\end{array}\right.
$$

If we use (4.16) to define an interaction between excitations, then (A.10) tells us that the excitations interact via hardcore exclusion with an activity $\zeta(\varepsilon)$.

In the region of convergence of the C.L.H.T. expansion

$$
|\zeta(\varepsilon)| \leqq e^{-k|\varepsilon|}
$$

where $k$ is a large constant and $|\varepsilon|$ denotes the cardinality of $\varepsilon$, minus the cardinality of $\pi(\varepsilon)$. The bound (A.11) ensures the convergence of the Mayer series for the gas of excitations.

Thanks to the factorization (A.9) we can give an explicit formula for the pressure of the gas of excitations defined as in Sect. 4.2:

$$
t P_{[0, t]}=t \ln z_{\omega}\left(c_{k+1}\right)+\sum_{\varepsilon} a_{T}(\varepsilon) \prod_{\varepsilon \in \varepsilon} \zeta(\varepsilon)
$$

where $\varepsilon$ denotes a collection of excitations. By comparing (4.19) and (A.12) one easily sees that only clusters of excitations, $\varepsilon$, whose projection onto the time axis contains $[0, t]$ contribute to the second term on the right-hand side of (A.12), $P_{\text {int }}$.

From (4.21) and (A.12) it follows that

$$
m\left(S_{1}\right)=-\ln z_{\omega}\left(c_{k+1}\right)+0\left(\zeta\left(\varepsilon_{\min }\right)\right)
$$

where $\varepsilon_{\min }$ is the excitation with the highest activity, and

$$
\mu\left(S_{1}\right)=0\left(\frac{1}{t} \ln \zeta\left(\varepsilon_{[0, t] \min }\right)\right),
$$

where $\varepsilon_{[0, t] \min }$ is the excitation with $\pi(\varepsilon) \supset[0, t]$ having the highest activity.

Using (A.7-14) one can prove the behaviour (4.20) for all our models with precise estimates on $m\left(S_{1}\right), \mu\left(S_{1}\right)$.

We discuss in some detail the case of the $\phi^{4}$ model in two dimensions with the help of some self-explanatory drawing. The results for the other models summarized in Table 1 (Sect. 4.2) are obtained in a similar way, using Table 2 (Appendix 1). In the $\phi_{2}^{4}$ model the contribution, $z_{\omega}(\langle x y\rangle)$, of a link to $Z(\emptyset)$ is given by the sum of two terms: the first one corresponds to the situation in which $\langle x y\rangle$ is only in the $v^{\omega}$ polymer and the second one to the situation in which it is also the support of an $X$-polymer.

As a result we can estimate for $\beta, \lambda$ large, $\beta / \lambda$

$$
z_{\omega}(\langle x y\rangle) \sim e^{-2 \beta}\left(1+0\left(\frac{\beta}{\lambda^{1 / 2}}\right)\right) \sim e^{-2 \beta\left(1+0\left(\lambda^{-1 / 2}\right)\right)} .
$$


The leading excitations are given by configuration like

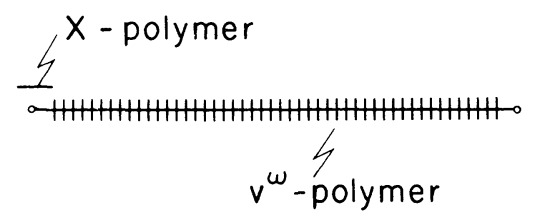

hence $\zeta\left(\varepsilon_{\min }\right) \sim 0(\beta / \lambda)$. The leading contribution to $P_{\text {int }}$ comes from configurations like

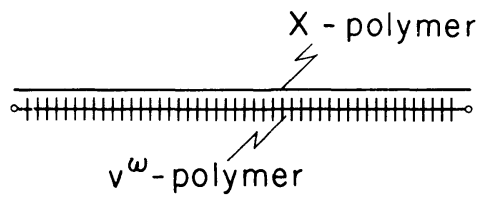

hence $\zeta\left(\varepsilon_{[0, t] \min }\right) \sim 0(\beta / \lambda)^{t}$.

Therefore, for the $\phi^{4}$ model, for $\beta$ large, $\beta / \lambda^{1 / 2}$ small, $\beta \gg|\ln (\beta / \lambda)|$,

$$
\begin{aligned}
& m\left(S_{1}\right)=2 \beta\left(1+0\left(\lambda^{-1 / 2}\right)\right)+0\left(\frac{\beta}{\lambda}\right), \\
& \mu\left(S_{1}\right)=0\left(\left|\ln \frac{\beta}{\lambda}\right|\right) .
\end{aligned}
$$

Remark $A$.2. For $\lambda=\infty$, the $\phi_{2}^{4}$ model reduces to the 2- $d$ Ising model.

In this limit there are no $X$-polymers, hence the leading excitations are given by configurations like

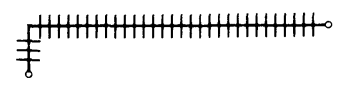

and the leading contributions to $P_{\text {int }}$ come from configurations like

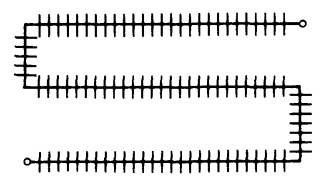

Hence for the (Ising)-model

$$
m\left(S_{1}\right)=2 \beta+0\left(e^{-\beta}\right), \quad \mu\left(S_{1}\right)=0(4 \beta) .
$$

Therefore we see that the presence of a continuous field $\phi$ in $\phi_{2}^{4}$ completely changes the upper gap $\mu\left(S_{1}\right)$.

\section{References}

1. Fröhlich, J., Marchetti, P. A.: Magnetic monopoles and charged states in four-dimensional, abelian lattice gauge theories. Europhys. Lett. 2, 933-940 (1986)

2. Fröhlich, J.: in, Progress in gauge theory. (Cargèse 1979), G. 't Hooft et al. (eds.). New York:

Plenum Press 1980 
3. t'Hooft, G.: Nucl. Phys. B138, 1 (1978)

4. Bricmont, J., Fröhlich, J.: Nucl. Phys. B25 [FS 13], 517 (1985); Commun. Math. Phys. 98, $553(1985$

5. Marino, E. C., Swieca, J. A.: Nucl. Phys. B170 [FS 1], 175 (1980); Marino, E. C., Schroer, B., Swieca, J.A.: Nucl. Phys. B200[FS 4], 473 (1982)

6. Borchers, H.J.: Commun. Math. Phys. 1, 57 (1965); Buchholz, D., Fredenhagen, K.: Commun. Math. Phys. 84, 1 (1982)

7. See e.g.: Solitons and particles, Rebbi, C. et al. (eds.) Singapore: World Scientific 1984

8. Fröhlich, J.: In: Invariant wave equations. Lecture Notes in Physics, Vol. 73. Berlin, Heidelberg, New York: Springer 1978

9. Fröhlich, J.: Commun, Math. Phys. 47, 269 (1976)

10. See e.g. Kadanoff, L.P., Ceva, H.: Phys. Rev. B11, 3918 (1971); Wegner, F.: J. Math. Phys. 12, 2259 (1971); 't Hooft, G.: In [3]; Mack, G., Petkova, V. B.: Ann. Phys. 123, 442 (1979); 125, 117 (1980); Kogut, J.: In: Recent advances in field theory and statistical mechanics. (Les Houches 1982) Zuber, J.-B. et al. (eds.) Amsterdam: North Holland 1984; Fröhlich, J., Spencer, T.: In: Scaling and self-similarity in physics, PPh 7, Basel, Boston: Birkhäuser 1983

11. Osterwalder, K., Schrader, R.: Commun. Math. Phys. 31, 33 (1973), 42, 281 (1975); Glaser, V.: Commun. Math. Phys. 37, 257 (1974); Fröhlich, J., Osterwalder, E., Seiler, E.: Ann. Math. 118, 461 (1981)

12. Seiler, E.: Gauge theories as a problem of constructive quantum field theory and statistical mechanics. Lecture Notes in Physics Vol. 159. Berlin, Heidelberg, New York: Springer 1982

13. Fredenhagen, K., Marcu, M.: Commun. Math. Phys. 92, 81 (1983)

14. Wilczek, F.: Phys. Rev. Lett. 48, 1144 (1982); 49, 957 (1982); Wilczek, F., Zee, A.: Phys. Rev. Lett. 51, 2250 (1982); Wu, Y.S.: Phys. Rev. Lett. 53, 111 (1984)

15. See e.g. Thouless, D.J., Wu, Y.S.: Phys. Rev. B31, 1191 (1985)

16. Fröhlich, J., Marchetti, P.A.: In preparation

17. Marchetti, P.A.: Ph. D. Thesis, S.I.S.S.A. Trieste 1986

18. Marchetti, P.A.: Particle analysis of soliton sectors in massive lattice field theories (in preparation)

19. Kennedy, T., King, C.: Commun. Math. Phys. 104, 327 (1986)

20. See e.g. Jackiw, R., Rebbi, C.: Phys. Rev. D13, 3398 (1975), and other papers in [7]

21. Fröhlich, J.: In: Renormalization theory. Erice 1975. Velo, G., Wightman, A. S. (eds.) NATO Advanced Study Institutes Series C23, 1976

22. Fröhlich, J.: In: Current problems in elementary particle and mathematical physics, Schladming 1976. Acta Phys. Austriaca [Suppl.]XV. Berlin, Heidelberg, New York: Springer 1976

23. Marra, R., Miracle-Solé, S.: Commun. Math. Phys. 67, 233 (1979)

24. Buchholz, D., Fredenhagen, K.: In [6]

25. Borgs, C., Nill, F.: No Higgs mechanism in scalar lattice Q.E.D. at large electromagnetic coupling. Preprint MPI-PAE/Pth 79/85

26. Buchholz, D.: Commun. Math. Phys. 85, 49 (1982)

27. Balaban, T., Brydges, D., Imbrie, J., Jaffe, A.: Ann. Phys. 158, 281 (1985); Brydges, D.: In: Critical phenomena, random systems, gauge theories. Osterwalder, K., Story, R. (eds.) (Les Houches 1984). Amsterdam: North Holland 1986

28. Steinmann, O.: Ann. Phys. 157, 232 (1984)

Communicated by A. Jaffe

Received March 27, 1987 
